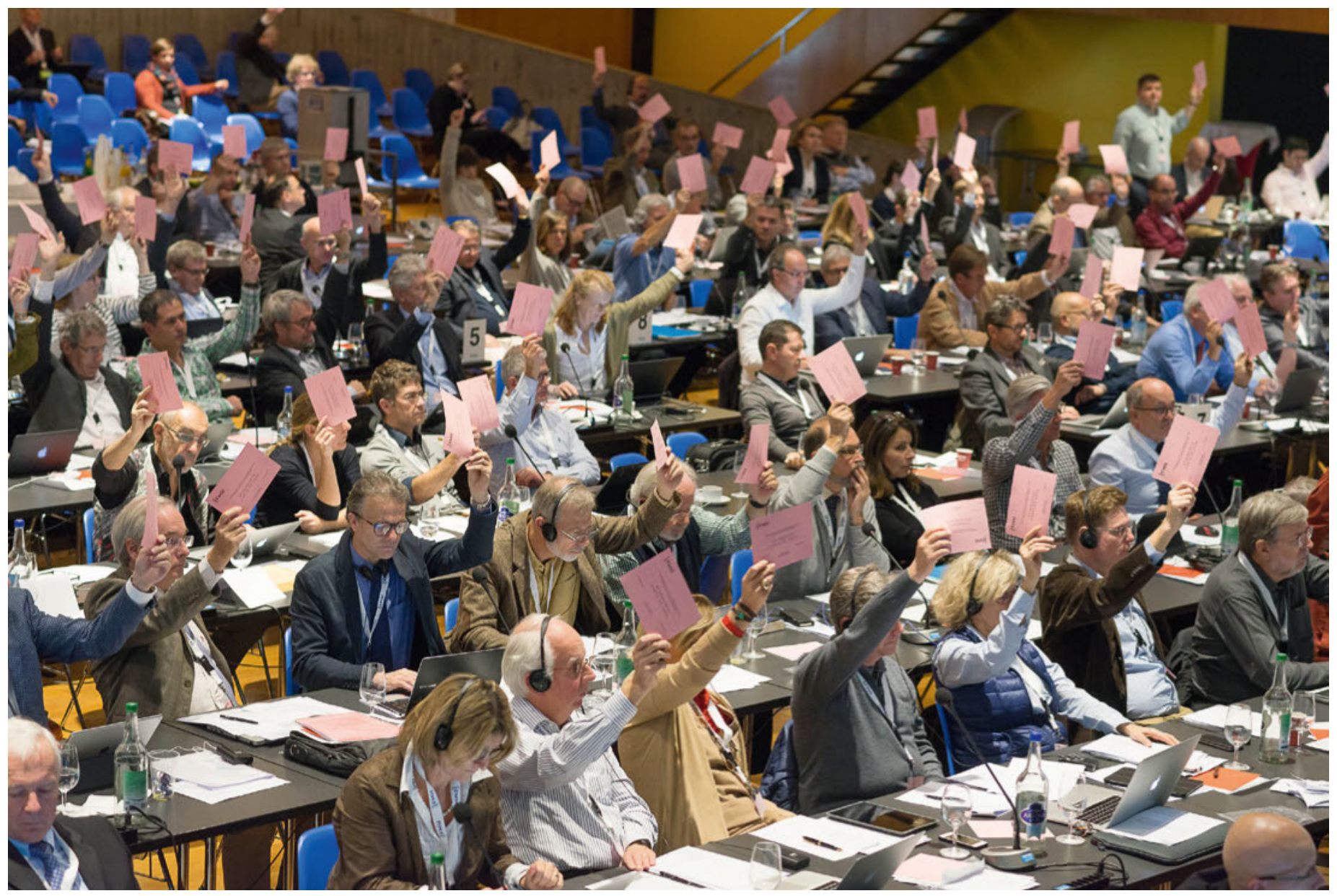

Konsens an der ÄK - die Delegierten entscheiden über ein FMH-Geschäft.

\title{
Beschlussprotokoll der zweiten Ärztekammer 2016
}

\section{Monika Henzen}

Leiterin Abteilung Zentrales Sekretariat

Anmerkung der Protokollführerin: Aus Gründen der Lesbarkeit werden die Traktanden nach Massgabe der Nummerierung und nicht in der Reihenfolge der Diskussion aufgeführt.

\section{Begrüssung, Mitteilungen, Bestellung des Büros}

Jürg Schlup/Präsident FMH begrüsst die Delegierten und die eingeladenen Gäste zur zweiten Ärztekammer
2016. Nach den üblichen organisatorischen Mitteilungen wird das Büro bestellt. Dieses besteht aus dem Präsidenten, den Vizepräsidenten, der Generalsekretärin, Olaf Wetter/Leiter des Stimm- und Wahlbüros sowie den nachfolgenden Stimmenzählenden, welche grossmehrheitlich mit 2 Gegenstimmen gewählt wurden: Christian Bernath, Karl Kothbauer, Lukas Meier, Marcel Stampfli, Jürg Lareida, Josef Emil Brandenberg, Brigitte Muff, Michael Zellweger, Karl-Olof Lövblad und Daniel Schröpfer. 
Anne-Geneviève Bütikofer/GS FMH weist darauf hin, dass die ÄK am 28.10.2015 im Rahmen der Budgetstabilisierungsmassnahmen entschieden hat, für die ÄKSitzungen nur noch Beschlussprotokolle zu verfassen. Die Delegierten haben jedoch die Möglichkeit, nach schriftlicher Anfrage beim Präsidenten FMH und bei der Generalsekretärin FMH das Audiofile zum angefragten Traktandum anzufordern.

\section{Traktandenliste}

Antrag:

Genehmigung der als Tischvorlage vorliegenden Traktandenliste.

Beschluss:

Die Traktandenliste wird einstimmig genehmigt. Gemäss Art. 11 Abs. 3 GO legt die ÄK zu Beginn jeder Sitzung die Zeit fest, nach deren Ablauf weder Beschlüsse gefasst noch Wahlen vollzogen werden dürfen. Der Präsident schlägt als Tagungsende 17.30 Uhr vor.

Antrag Festlegung Tagungsende

Als Tagungsende wird 17.30 Uhr festgelegt. Nach 17.30 Uhr dürfen weder Beschlüsse gefasst noch Wahlen vollzogen werden.

Beschluss:

Der Antrag wird mit 150 Ja, 2 Nein und 1 Enthaltung angenommen.

Weiter stellt der Vorsitzende zwei Ordnungsanträge betreffend Redezeit und Einreichung der Anträge.

Gemäss Art. 11 Abs. 7 GO kann auf Vorschlag aus der Kammer oder des Vorsitzenden die Redezeit allgemein beschränkt werden. Aufgrund der Traktanden und der zur Verfügung stehenden Zeit wird eine Redezeitbeschränkung von zwei Minuten beantragt.

Ordnungsantrag 1-Redezeitbeschränkung:

Für die Behandlung der Traktanden der heutigen ÄK gilt eine Redezeitbeschränkung von 2 Minuten für Einzelredner. Keine Beschränkung gilt für den Präsidenten oder seinen Stellvertreter der jeweiligen stimm- oder antragsberechtigten Ärzteorganisation sowie für den Sprecher des ZV.

Beschluss:

Der Antrag wird mit 150 Ja und 2 Enthaltungen angenommen.

Art. 11 Abs. 5 der GO sieht vor, dass jeder Delegierte, jedes ZV-Mitglied und der Generalsekretär das Recht haben, Anträge zu den zur Diskussion stehenden Traktanden zu stellen. Anträge sind dem Vorsitzenden schriftlich vor oder während der Sitzung oder ausnahmsweise mündlich einzureichen.

Ordnungsantrag 2 - Schriftliche Einreichung der Anträge:

Abänderungsvorschläge und Anträge sind dem Vorsitzenden schriftlich einzureichen. Mündliche Anträge werden nicht entgegengenommen und sind ungültig.

Beschluss:

Der Antrag wird mit 109 Ja, 15 Nein und 6 Enthaltungen angenommen.

\section{Eröffnungsreferat des Präsidenten}

In seinem Eröffnungsreferat geht Jürg Schlup/Präsident FMH auf die kürzlich publizierte Studie von Prof. Beck

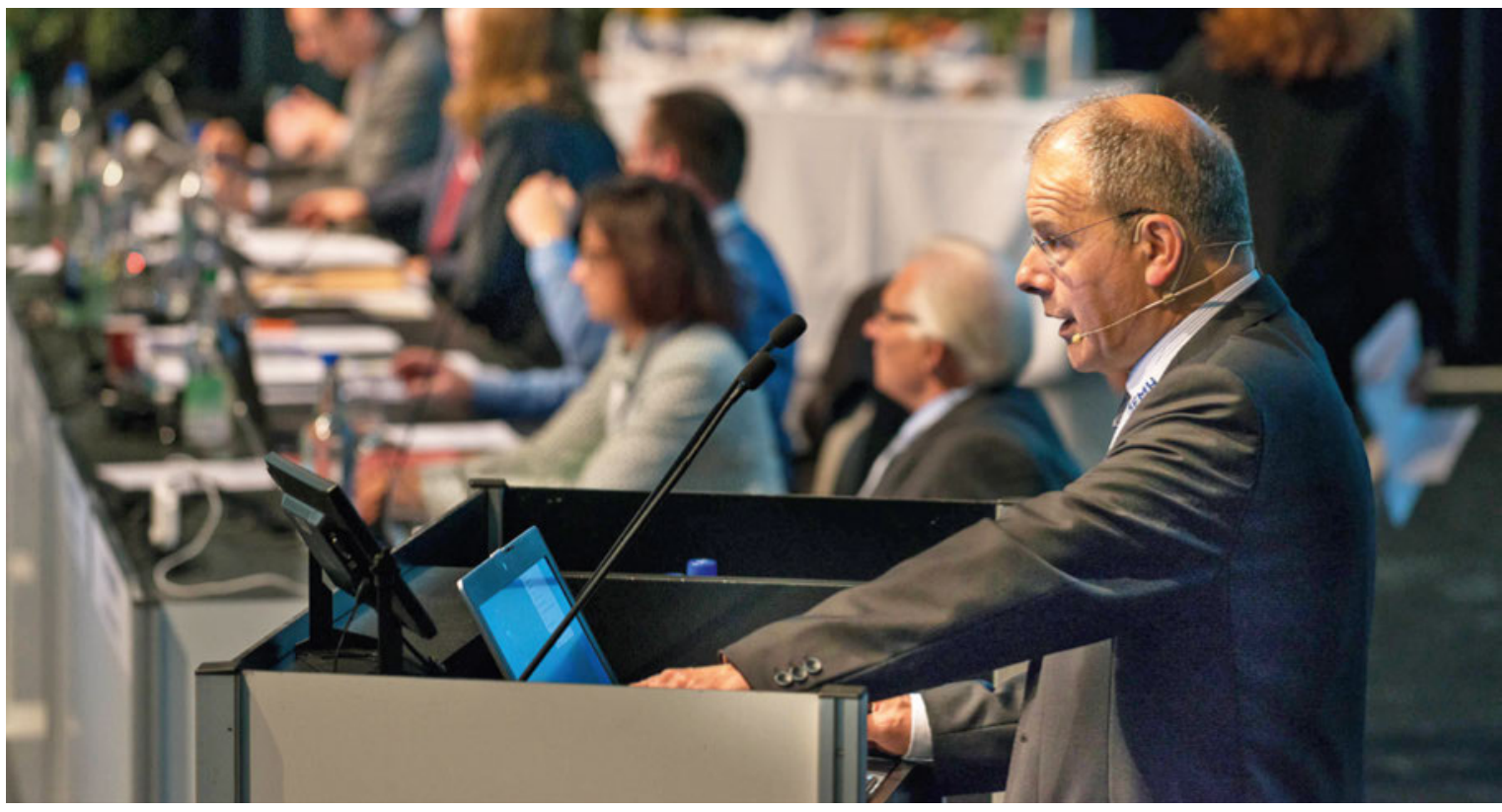

FMH-Präsident Dr. med. Jürg Schlup führt zielstrebig durch die zweite Ärztekammer dieses Jahres. 
und Dr. Telser im Auftrag der CSS-Krankenversicherung "Kosten und Nutzen von medizinischen Behandlungen am Lebensende» ein. Die Resultate sind bemerkenswert auch für die Ärzteschaft. Die Behandlungspraxis am Lebensende ist angemessen! Die demografische Alterung ist ein entscheidender Kostentreiber. Die vielfach zitierte Behauptung, wonach betagte Menschen in ihren letzten Monaten aus ökonomischen Interessen noch überbehandelt werden, wird hier widerlegt. Grossmehrheitlich entsprechen die Behandlungskosten am Lebensende dem, was die Schweizer Bevölkerung als vernünftig empfindet.

Wichtige Herausforderungen und Themen haben die FMH auch im 2016 begleitet. Recht einseitig berichten die Medien über die steigenden Kosten in der Krankenversicherung. Ein Vergleich bei den übrigen Sozialversicherungen in den letzten 25 Jahren zeigt, dass die KVG-Ausgaben völlig parallel mit den Sozialversicherungsausgaben verlaufen.

Immer mehr Kantone schränken die ärztliche Schweigepflicht ein. Die FMH verteidigt das Berufsgeheimnis und plädiert für ein Vertrauensverhältnis zwischen Arzt und Patienten. Die kantonalen Ärztegesellschaften können jederzeit bei geplanten Gesetzesänderungen auf die Unterstützung der FMH zählen.

Die Schweizerische Hochschulkonferenz (SHK) überprüft zurzeit das Auswahlverfahren (Numerus Clausus) der medizinischen Fakultäten Basel, Bern, Fribourg und Zürich. Die FMH empfiehlt, die heute schwergewichtig naturwissenschaftlich-mathematische PCPrüfung mit einem Assessment in Empathie und Sozialkompetenz zu ergänzen.

Seit Jahren lobbyiert die FMH für wirksame Massnahmen gegen den Nachwuchsmangel. Erfreulicherweise hat das eidgenössische Parlament in der letzten Herbstsession dem Antrag von BR Schneider-Ammann zugestimmt und für die Schaffung neuer Medizinstudienplätze ab 2017 CHF 100 Mio. bewilligt. Die Universität Zürich wird ihre Medizinstudienplätze bereits ab August 2017 um 72 Plätze, die Universität Bern ab August 2018 mit 100 Plätzen erhöhen. Mit den bereits erfolgten Erhöhungen werden 2020 in der Schweiz wieder ebenso viele Ärzte diplomiert werden wie Ende der 1970er Jahre. Diese Ausbildungsanstrengung wirkt sich aber erst zu Beginn der 2030er Jahre voll aus. Dies ist mit ein Grund, weshalb die FMH gegen eine harte Umsetzung der Masseneinwanderungsinitiative ist. Die Schweiz ist im Gesundheitswesen auf die bilateralen Verträge und ein gutes internationales Umfeld angewiesen.

Die bisherige Zulassungssteuerung von Ärztinnen und Ärzten mit den Qualitätskriterien «drei Jahre Berufstätigkeit an anerkannter Weiterbildungsstätte» (gemäss Art. 55a KVG) ist seit Mitte 2013 in Kraft. Mit ei- nem dringlichen Bundesgesetz wurde diese Regelung heuer um weitere drei Jahre verlängert. Gleichzeitig hat das Parlament mit je einem Vorstoss der Gesundheitskommissionen beider Kammern den Bundesrat mit der Ausarbeitung eines Berichts bis Ende dieses Jahres beauftragt. Dieser Bericht wie auch ein anschliessend neu auszuarbeitendes Gesetz sollen aufzeigen, wie der Bund künftig die Zulassung ambulant tätiger Ärztinnen und Ärzte einschränken will. Die FMH findet den vom Parlament im Sommer 2016 vorgeschlagenen Lösungsansatz ungeeignet und empfiehlt eine transparente und auf Qualitätskriterien beruhende Zulassungssteuerung. Folgende nationale Qualitätskriterien sollten kumulativ erfüllt sein:

- Nachweis von mindestens drei Jahren klinischer Tätigkeit in der für die Zulassung beantragten Fachdisziplin an einer anerkannten Wirkungsstätte in der Schweiz;

- Nachweis der für die Tätigkeitsregion erforderlichen Sprachkompetenzen in Form einer in der Schweiz abgelegten Sprachprüfung;

- Nachweis eines Studiums der Humanmedizin von mindestens sechs Jahren Dauer (Vollzeit) oder mindestens 5500 Stunden theoretischen und praktischen Unterricht;

- Den periodischen Nachweis eines Fortbildungsdiploms.

\section{Revision der ambulanten Tarifstruktur (Projekt TARCO)}

Jürg Schlup/Präsident FMH erinnert an den Entscheid der Urabstimmung, welche sämtliche Anträge der ÄK abgelehnt hat. Aufgrund dieses Ergebnisses hat die FMH am 30.6.2016 beim BAG keine Tarifstruktur eingereicht. Eine Einigung der Tarifpartner auf eine revidierte Tarifstruktur innerhalb der von BR Berset verlängerten Frist bis Ende Oktober 2016 wird ebenfalls nicht zustande kommen. Allein für die FMH wäre ein interner Konsens mit ihren rund 90 angeschlossenen Organisationen innerhalb einer Frist von vier Monaten nicht zu erreichen. Dem Vorsteher des EDI wurde bereits kommuniziert, dass die FMH bis Ende Oktober 2016 weder eine revidierte Tarifstruktur noch Vorschläge für eine Anpassung der aktuell gültigen Tarifstruktur TARMED einreichen wird. Da H+ den Rahmenvertrag mit santésuisse gekündigt hat, drohte per 1.1.2017 ein tarifstrukturloser Zustand. Die vier Tarifpartner konnten sich auf eine einjährige Verlängerung betreffend Gültigkeit des TARMED 1.08 BR bis 31.12.2017 einigen.

Nach dem Entscheid der Urabstimmung hat der ZV FMH alle Präsidenten der angeschlossenen Ärzteorga- 
nisationen am 18.7.2016 zu einem Austauschtreffen eingeladen. Eine Mehrheit der Anwesenden hat sich an dieser Konsultativbefragung für eine Weiterführung/ Nachbesserung der Revision auf Basis der ats-tms-Version 1.0 Tarifstruktur ausgesprochen. Aufgrund dieses Resultats hat das Departement Ambulante Versorgung und Tarife ein Grobkonzept und einen Vorgehensvorschlag zur Nachbesserung der revidierten Nomenklatur erstellt.

Urs Stoffel/ZV FMH und Verantwortlicher des Departementes Ambulante Versorgung und Tarife informiert über den aktuellen Stand des Projekts TARCO. Der ZV und die DV haben ihr Einverständnis zum Grobkonzept und Vorgehen erteilt und das Departement anfangs September 2016 mit dem Projekt TARCO mandatiert. Dieses Projekt wird als internes Projekt aufgegleist; die Tarifpartner werden nach Abschluss der internen Revisionen einbezogen. Das Detailkonzept wird bis zur DV vom 26.1.2017 ausgearbeitet. Parallel dazu wird eine Arbeitsgruppe, bestehend aus Vertretern der Dachverbände, die bisher ungeklärten und tarifkapitelübergreifenden Problemstellungen wie Dignitäten vor einer detaillierten Nachbesserung auf Kapitelebene zu lösen versuchen. Mit den Fachgesellschaften und Dachverbänden werden schriftliche Vereinbarungen getroffen. Für die FMH ist es zentral, die Anliegen und Interessen der Ärzteschaft für eine sachgerechte und faire Abgeltung der ambulanten ärztlichen Leistungen in den Vordergrund zu stellen. Die Kostenneutralität, die bei der Urabstimmung mit $89 \%$ abgelehnt wurde, wird nicht mehr angestrebt. Der Fokus wird auf eine betriebswirtschaftliche und sachgerechte Revision vornehmlich der praxisambulanten Leistungen gelegt. Die Nachbesserungen sollen unabhängig von Zeitlimiten und notwendigen Nachbesserungen vorerst FMH-intern erfolgen. Die Wahrung der Tarifautonomie soll erhalten bleiben. Die Vernetzung und Zusammenarbeit mit den Fachgesellschaften und Dachverbänden ist wichtig. Die ärztlichen Leistungen (AL) im spital-ambulanten Bereich werden ebenfalls überprüft. Einschränkend ist hier die fehlende bzw. nicht umfassende Zahlenbasis der spital-ambulanten Leistungen (Mengengerüste).

Die DV wird am 26.1.2017 über das Detailkonzept beraten. Bevor das Projekt TARCO überhaupt gestartet wird, ist die Problemstellung der Dignitäten zwingend vorher zu lösen.

2.1 Antrag VSAO: Den spital-ambulanten Bereich im Projekt TARCO nicht vergessen - Rückzug

Angelo Barille/VSAO hält fest, dass bei der Projektpräsentation an der DV vom 7.9.2016 ausschliesslich der praxis-ambulante Bereich erwähnt wurde. Dem VSAO ist es ein Anliegen, dass im FMH-internen Projekt TARCO der spital-ambulante Bereich mitgeplant wird und die Tarifautonomie in diesem Bereich ebenso erhalten bleibt. Die FMH muss gewährleisten, dass es künftig einen und nicht mehrere Tarifsysteme für den ambulanten Bereich gibt, weil sonst Fehlanreize und eine Unterfinanzierung im spital-ambulanten Bereich drohen. Für den VSAO ist nach den vorherigen Ausführungen von Urs Stoffel/ZV FMH sichergestellt, dass der spital-ambulante Tarif im Projekt TARCO ebenfalls miteinbezogen wird. Aus diesem Grunde wird der Antrag zurückgezogen.

Urs Stoffel/ZV FMH dankt für das Vertrauen und versichert dem VSAO, dessen Anliegen in das Projekt TARCO aufzunehmen.

\section{3. Änderungen in Statuten, Geschäfts- und Standesordnung und GPK-Reglement}

\section{1 Änderung im Reglement der Geschäfts- prüfungskommission der FMH (GPK)}

\subsubsection{Antrag GPK: Änderung Art. 5.2: Amtsdauer, Ersatzwahlen}

Adrian Sury/Präsident GPK führt aus, dass sich die GPK gemäss Reglement alle zwei Jahre neu konstituiert, und zwar mit einer Amtsdauer von zwei mal zwei Jahren im gleichen Ressort. Mit dieser maximalen Amtsdauer wird die Arbeit der GPK eingeschränkt, da bei jedem Wechsel viel Know-how verloren geht. Im heutigen Reglement ist ein Wechsel spätestens nach vier Jahren zwingend. Die GPK wünscht die Streichung des zweiten Satzes von Art. 5.2. Abs. 1. Damit würde die gleiche Ressortzuständigkeit zugelassen, wie sie im ZV gilt. Unverändert bleibt die Begrenzung auf maximal drei Amtsdauern von je vier Jahren nach Art. 53 Abs. 1 der Statuten der FMH.

Antrag GPK:

Abschaffung des zweiten Satzes im Abs. 1 des Art. 5.2 des GPK Reglements und Ersatz von "Amtsdauer, Ersatzwahlen" durch "Überprüfung der Funktionen, Ersatzwahlen" im Titel von Art. 5.2: "5.2 Überprüfung der Funktionen, Amtater, Ersatzwahlen"

${ }^{1}$ Im Rahmen der Amtsdauer von vier Jahren gemäss Art. 53 Abs. 1 der Statuten FMH werden die Funktionen innerhalb der GPK alle zwei Jahre neu vergeben. Đie maximale Amtstater für eine funktion beträgt viex fahre:

Beschluss:

Der Antrag wird mit 150 Ja, 5 Nein und 1 Enthaltung angenommen. 


\section{Budgets 2017 von SIWF, FMH und GPK}

\subsection{Budget 2017 SIWF}

Werner Bauer/Präsident SIWF geht einleitend auf die Gründe des negativen Budgets des SIWF 2017 ein. Das Defizit ist einerseits auf den starken Rückgang der Titelgesuche und andererseits auf die kostenintensiven IT-Projekte zurückzuführen. Weiter ist auch die Akkreditierung 2018, für welche das SIWF vom BAG das Mandat erhalten hat, mit hohen Kosten verbunden (2017 und 2018 mit je CHF 400000). Die Strukturveränderungen in den Spitälern haben auch Auswirkungen auf das e-Logbuch. Im Hinblick auf die interdisziplinären Weiterbildungsgänge ist das e-Logbuch entsprechend anzupassen. Mit der Erhöhung der Studienplätze in den kommenden Jahren wird auch der Anspruch an Steuerungsmöglichkeiten in der Aus- und Weiterbildung zunehmen. Das SIWF beschäftigt sich schon heute mit dieser Fragestellung.

Christoph Hänggeli/Geschäftsführer SIWF geht als erstes auf die zwei budgetrelevanten IT-Projekte ein. Beim e-Logbuch funktionieren alle Kernelemente gut. Alle 83 Weiterbildungsprogramme sind parametrisiert und bis heute haben sich bereits 16000 Benutzer registriert. Alle Weiterbildungsanforderungen werden online erfasst. Die Bearbeitung der Gesuche und der Standortbestimmungen erfolgt in einem elektronischen Workflow. Nicht zuletzt wegen den individuellen Wünschen der Fachgesellschaften ist jedoch die Wartung und Weiterentwicklung des Systems nicht mehr gewährleistet. Die Einführung neuer Module musste gestoppt werden und eine Analyse des Systems wurde in Auftrag gegeben. In erster Priorität muss die Komplexität des Systems vereinfacht und die Usability verbessert werden. Die entsprechenden Kosten von CHF 500000 sind im Budget 2017 reserviert.

Die e-Fortbildung funktioniert technisch auf einer neuen Plattform und ist jetzt auch für Mobile und Tablets geeignet. Die Benutzerfreundlichkeit wurde optimiert. Der Betrieb, die Wartung und Weiterentwicklung sind im Unterschied zum e-Logbuch gewährleistet. In einer weiteren Ausbaustufe ist ein zentrales Register für alle Veranstaltungen geplant. Angesichts der Budgetsituation und wegen der Priorisierung des e-Logbuchs wurde dieser Ausbau vorläufig sistiert.

Der positive Rechnungsabschluss 2015 war auf die zusätzlichen nicht budgetierten Titelerteilungen zurückzuführen. Demgegenüber droht jetzt für 2016 ein Rückgang der erteilten Titel um 30\%. Diese Entwicklung stellt das SIWF vor grosse Herausforderungen, auch wenn der Verlust beim gegenwärtigen Vermögensstand noch aufgefangen werden kann. 2017 rechnet das SIWF mit einem Verlust von CHF 867000. Neben dem e-Logbuch bildet auch die Akkreditierung einen hohen Ausgabeposten. Das SIWF hat zusätzliche Massnahmen ergriffen, damit Aufwand und Ertrag mittelfristig wieder ausgeglichen sind. Zu erwähnen sind insbesondere die kostendeckenden Gebühren für die Zertifizierung und Visitation der Weiterbildungsstätten sowie im Bereich der Fortbildung.

\subsection{Budget 2017 FMH}

Emanuel Waeber/Leiter Abteilung Verwaltung und Finanzen präsentiert für die FMH ein ausgeglichenes Budget 2017. Ausgehend vom Auftrag der ÄK vom Oktober 2014, für die FMH auch in Zukunft ein finanzielles Gleichgewicht zwischen den getätigten Ausgaben und Projekten sowie den generierten Einnahmen zu gewährleisten, wirken sich die vom ZV nun ausgearbeiteten und von der ÄK genehmigten Budgetstabilisierungsmassnahmen auf das Budget 2017 positiv aus. Damit kann sichergestellt werden, dass die FMH auch in Zukunft ihre Kernaufgaben wahrnehmen kann. Die vor zwei Jahren beschlossene Ausgabenbremse zeigt, dass die Gesamthöhe der im Budget aufgeführten Ausgaben der FMH von damals CHF 22,7 Mio. die jeweiligen Einnahmen von CHF 23,3 Mio. nicht übersteigen darf. Das Budget 2017 erreicht diese Zielvorgabe. Das konsolidierte Budget 2017 weist ein Defizit von CHF 217000 auf (Verlust SIWF von CHF 867000 und Gewinn FMH von CHF 650000). Der Verlust des SIWF resultiert insbesondere aus dem Projektmehraufwand und den Akkreditierungskosten. Dank der Erhöhung der Mitgliederbeiträge von mehr als CHF 1 Mio. sowie der Auflösung vorhandener Rückstellungen von CHF 520000 kann die FMH ein ausgeglichenes Budget präsentieren.

Gemäss Forecast per 3. Quartal 2016 wird auf Ende 2016 ein positives Ergebnis erwartet. Dieses Resultat ist jedoch mit Vorsicht zu betrachten, da laufende Projektkosten sowie die Bildung allfälliger Rückstellungen für Projektrealisierungen noch nicht berücksichtigt sind.

\subsection{Budget 2017 GPK}

Emanuel Waeber/Leiter Abteilung Verwaltung und Finanzen hält fest, dass das Budget 2017 im Vergleich zu 2016 keine Abweichungen ausweist. Die Kostenrechnung ist aufgrund des geringeren Personalaufwands, der auf die Budgetstabilisierungsmassnahmen zurückzuführen ist, um rund CHF 35000 tiefer.

\section{Bericht GPK}

In seinem Bericht geht Adrian Sury/Präsident GPK auf die wesentlichen drei Punkte ein:

Finanzielle Situation des SIWF, Budgetstabilisierungsmassnahmen und Befindlichkeit im Generalsekreta- 


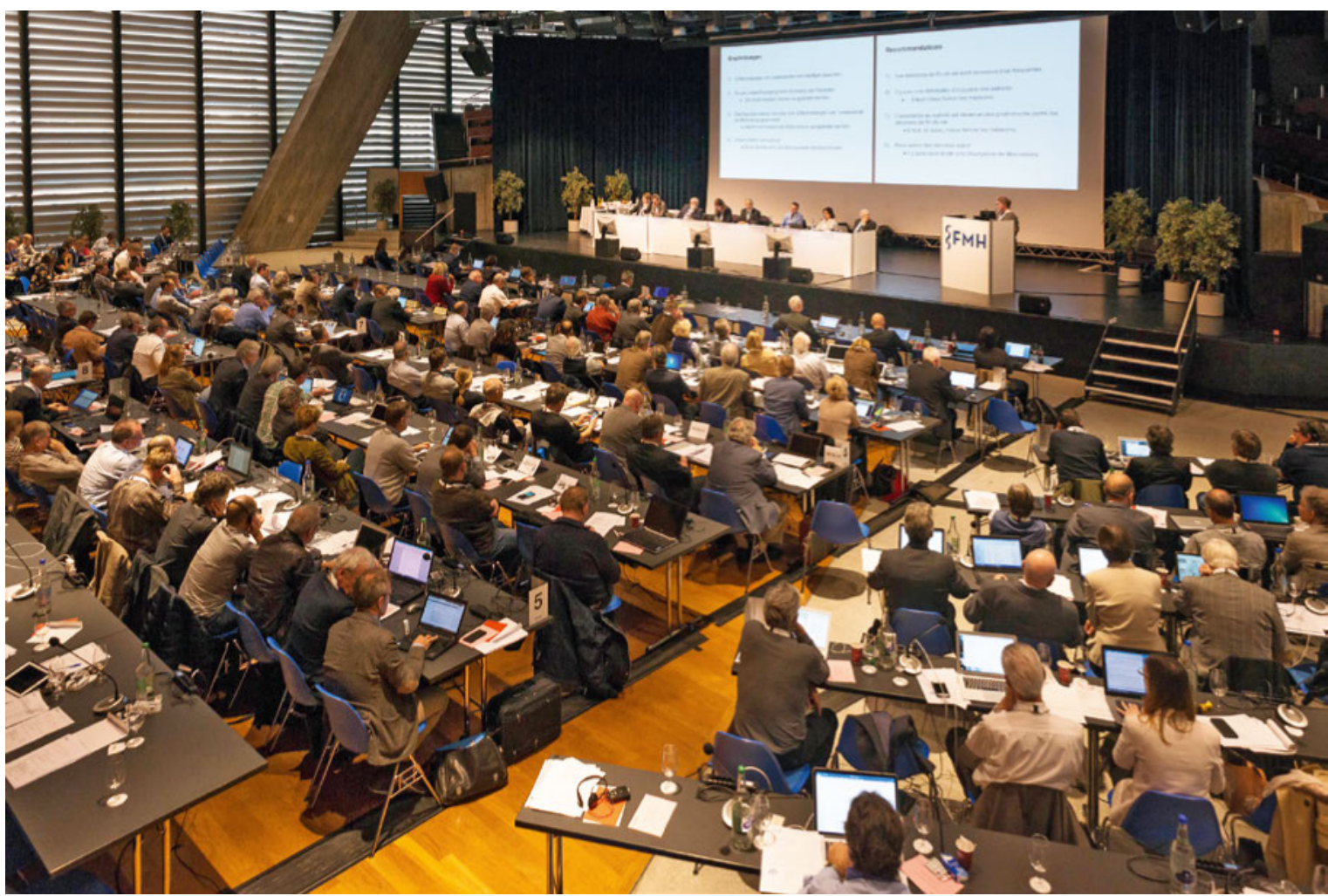

200 Delegierte zählt das Parlament der FMH.

riat. Aufgrund der besorgniserregenden Finanzlage empfiehlt die GPK dem SIWF eine bessere Planung ihrer Finanzen sowie der IT-Projekte. Das e-Logbuch führte zu grossen Verspätungen und Firmenwechseln. Aus Sicht der GPK ist eine Reevaluation 2017 angezeigt. Die Akkreditierungskosten sind hoch und eine Verteilung und Bildung von Reserven auf mehrere Jahre wäre möglich gewesen. Die GPK wollte ursprünglich das Budget des SIWF zurückweisen und der ÄK erst im Frühjahr zur Genehmigung unterbreiten.

Das von der FMH vorgelegte ausgeglichene Budget 2017 beweist, dass die FMH ihre Aufgabe gut gemacht hat. Die grossen Sparanstrengungen und die beschlossenen Massnahmen haben jedoch bei den Mitarbeitenden im Generalsekretariat Auswirkungen und die Befindlichkeit hat seiner Meinung nach gelitten.

Für die Anlagestrategie der FMH verdient der Leiter Abteilung Verwaltung und Finanzen ein grosses Lob. Es gibt nur wenige Firmen, die aktuell eine solche Rendite erzielen.

Jürg Schlup/Präsident FMH dankt der GPK für ihre Ausführungen. Einen grossen Dank spricht er ebenfalls der GS, den Kadern und allen Mitarbeitenden für ihr Engagement und ihre Unterstützung bei der Umsetzung der Budgetstabilisierungsmassnahmen aus.

Auf die Frage der im Budget 2017 aufgeführten Rückstellungen für das IPI von CHF 670000 stellt Anne-Ge- neviève Bütikofer/GS FMH klar, dass dieser Betrag für Praxisinformatik-Projekte beim Departement Digitalisierung und eHealth reserviert wurde. Da bis heute keine Projekteingaben erfolgten, muss dieser Betrag in der Buchhaltung als Rückstellung aufgeführt werden. Daniel Schröpfer/VSAO stellt aufgrund des präsentierten Budgets fest, dass bei den Mitarbeitenden viel gespart wurde. Er macht beliebt, dass bei einer positiven finanziellen Lage der FMH die Angestellten auch angemessen am Erfolg partizipieren können. Es ist dem VSAO ein Anliegen, dass die langjährigen Mitarbeitenden der FMH und das Back Office weiterhin gut funktionieren und einer Fluktuation vorgebeugt werden kann.

\subsection{Sonderbeitrag NAKO (NewIndex)}

Gemäss Urs Stoffel/ZV FMH ist die Datensammlung der praktizierenden Ärzte unerlässlich, um die Datenparität mit den Tarifpartnern zu sichern. Für die Weiterführung der Tarifrevision mit dem Projekt TARCO sind diese Daten wichtig. Gleichzeitig sind die Kantone der gekündigten Taxpunktwertanhänge und den drohenden Festsetzungsverfahren auf die Daten der NAKO zur Verteidigung der Taxpunktwerte angewiesen. Der Sonderbeitrag dient zur Basisfinanzierung der Infrastruktur und zur Sicherstellung des Betriebs der NewIndex AG und ermöglicht damit der Ärzteschaft einen lau- 
fenden Zugang zum NAKO-System als Grundlage zur gezielten Datenanalyse und Datenauswertung.

\section{Antrag ZV:}

Die FMH erhebt bei den Mitgliedern der Kategorien 1 und 2 einen zweckgebundenen Sonderbeitrag von CHF 40 zugunsten der Nationalen Konsolidierungsstelle NAKO.

\section{Beschluss:}

Der Antrag wird mit 150 Ja, 1 Nein und 9 Enthaltungen gutgeheissen.

\subsection{Weiterführung Sockelbeitrag 2017}

Nach den Entscheiden der ÄK im Rahmen der Budgetstabilisierungsmassnahmen hat die vom $\mathrm{ZV}$ beauftragte KPMG in ihrem Bericht Verbesserungspotenziale für den EMH-Verlag aufgezeigt. Nach der Diskussion dieser Potenziale hat der EMH-Verlag dem ZV entsprechende Vorschläge unterbreitet. Zwei dieser Vorschläge (Sockelbeitrag und Auslandportokosten) sind budgetrelevant und von der ÄK zu genehmigen. Beim ersten Vorschlag geht es um die Reduktion des 2011 von der ÄK beschlossenen Sockelbeitrags EMH/SMSR/ OMCT. Mit einer solchen Reduktion kann auch der Mitgliederbeitrag der Kategorien 1, 2, 3, 5, 6 reduziert werden.

Jürg Schlup/Präsident FMH informiert, dass die SMSR zu diesem Traktandum einen Antrag eingereicht hat und für die Beibehaltung des unveränderten Sockelbeitrags 2017 plädiert.
Der ZV beantragt im Budget 2017 ebenfalls die unveränderte Weiterführung des Sockelbeitrags. Pierre-Alain Schneider/Präsident SMSR ist einverstanden, den Antrag der SMSR zusammen mit dem Antrag des ZV zu behandeln.

Antrag ZV (zusammen mit identisch lautendem Antrag SMSR):

Genehmigung der vorgeschlagenen Weiterführung der Sockelabonnementsgebühren für 2017 mit CHF 75.00 ohne Kategorien 4 und 7 zugunsten von EMH: CHF 50 / SMSR CHF 20 / OMCT CHF 5.

Beschluss:

Der Antrag wird mit 108 Ja, 30 Nein und 8 Enthaltungen angenommen

\subsection{Antrag Ricardo Torriani/AGZ: Öffentlich- keitsarbeit}

Aus Sicht von Ricardo Torriani/AGZ werden die Anliegen der Ärzteschaft ungenügend vertreten. In der Öffentlichkeit und in den Medien werden Positionen gegen die Ärzteschaft publiziert, denen rascher widersprochen werden sollte. Er erachtet ein Ressort, welches sich mit Imagekampagnen und Öffentlichkeitsarbeit widmet, als zwingend. Seiner Meinung nach hat die FMH das Budget nicht gekürzt, um Geld zu sparen, sondern um es gezielter einzusetzen.

Jürg Schlup/Präsident FMH weist auf die von der ÄK 2014 beschlossene Ausgabenbreme hin. Eine Annahme dieses Antrags hätte eine Erhöhung der Mitgliederbei-

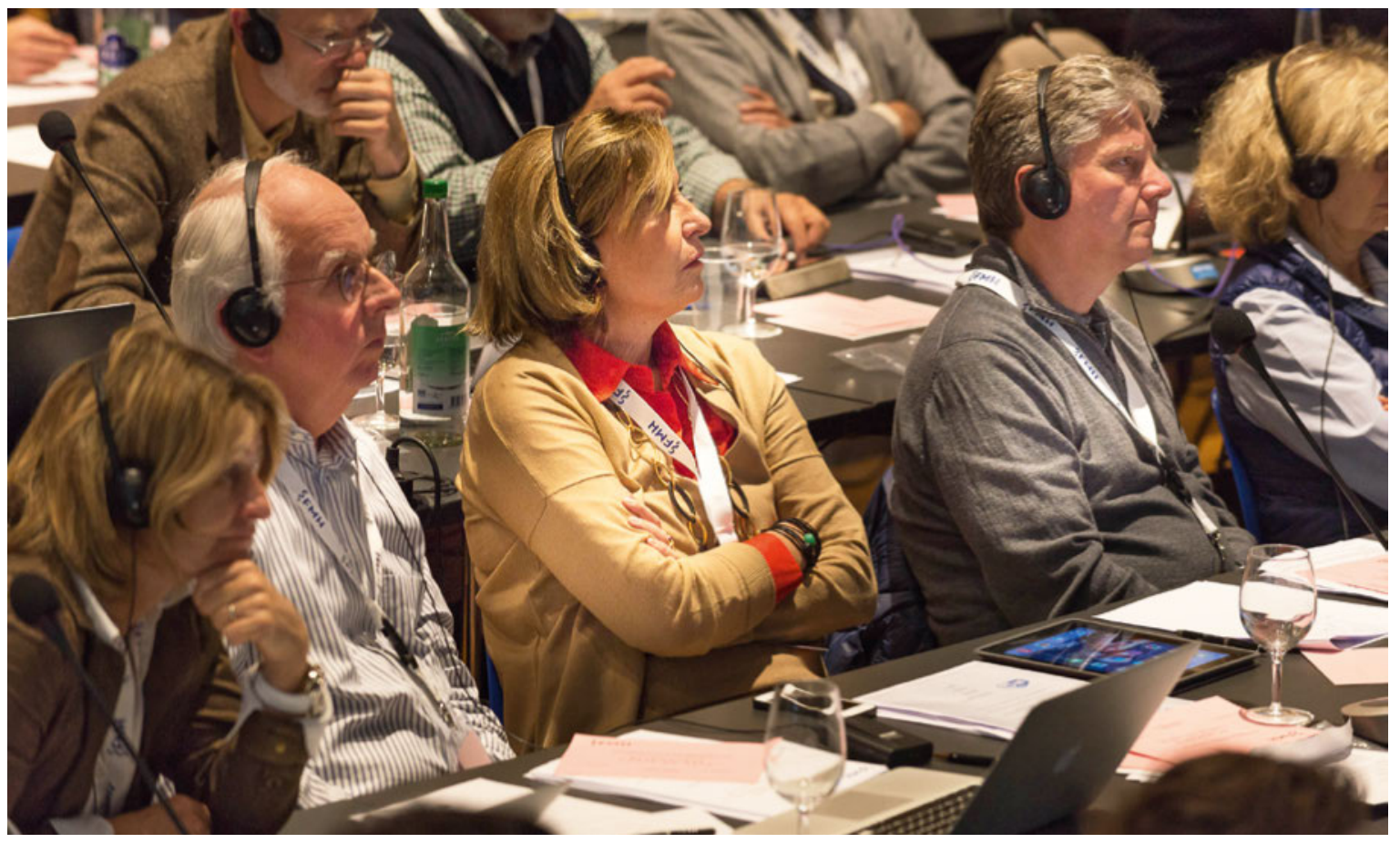

Interessiert lauschen diese Delegierten einem Votum. 
träge zur Folge. Nach ihm ist die FMH bereits heute in den Online- und Printmedien regelmässig vertreten. Eine entsprechende Statistik kann sowohl über Google wie auch über einen Mediensuchdienst abgefragt werden. Die FMH wird zudem regelmässig vom Parlament zu Hearings eingeladen - dieses Jahr beispielsweise fünf Mal. Eine Vor- und Nachbearbeitung der Sessionen erfolgt regelmässig. Wenn die ÄK eine grössere Präsenz wünscht und den Antrag Torriani unterstützt, sind die Mitgliederbeiträge für die Kategorie 1 und 2 um CHF 40 zu erhöhen.

Die Voten zeigen, dass es der FMH in den letzten Jahren gelungen ist, sich besser zu positionieren und den Bekanntheitsgrad zu steigern. Die Delegierten sprechen sich für mehr Qualität als Quantität aus. Die Mehrheit der Anwesenden unterstützt den Antrag Torriani nicht, weil sie überzeugt sind, dass mit mehr Öffentlichkeitsarbeit und einem neuen Ressort das Image in der Öffentlichkeit nicht verbessert werden wird.

Antrag Torriani:

Der Vorstand der FMH begründet ein Ressort Öffentlichkeitsarbeit. Dieses Ressort ist dem Präsidenten gemäss der aktuellen Verteilung im Vorstand der FMH unterstellt. Es wird ein Budget für die Öffentlichkeitsarbeit von CHF 1 Mio. jährlich bereitgestellt. Es werden für den Aufbau von Kampagnen professionelle Werbeberater beigezogen. Der Präsident erstattet an jeder Ärztekammer über die Aktivitäten Bericht.

Beschluss:

Der Antrag wird mit 113 Nein, 30 Ja und 10 Enthaltungen abgelehnt.

4.7 Antrag Aargauischer Ärzteverband: Finanzierung juristisches Vorgehen Bearbeitungsreglement gemäss Art. 30 c KVV

Jürg Lareida/Präsident Aargauischer Ärzteverband erklärt, dass der Aargauische Ärzteverband überzeugt ist, dass die Auslegung des Gesetzesartikel weit über das Ziel hinausschiesst und die Daten mit einem ungenügenden Schutz der Leistungserbringer und Patienten direkt zur Steuerung der Kosten im Gesundheitswesen verwendet werden. Mit diesem Vorgehen wird die Therapiefreiheit stark eingeschränkt und die heutige Medizin wird sich zur Staatsmedizin entwickeln. Der Aargauische Ärzteverband fühlt sich bei der im November zu startenden Datenerhebung MARS seinen Mitgliedern gegenüber verpflichtet, juristische Abklärungen vorzunehmen und entsprechend notwendige Schritte in die Wege zu leiten.

Antrag Aargauischer Ärzteverband:

Im Budget 2017 seien CHF 100000 für die Bekämpfung von MARS einzustellen.
Beschluss:

Der Antrag wird mit 123 Ja, 12 Nein und 23 Enthaltungen angenommen.

\subsection{Mitgliederbeitrag 2017}

Für 2017 ergeben sich folgende Mitgliederbeiträge:

Kategorie 1: $\quad$ CHF 825

Kategorie 2: $\quad$ CHF 825

Kategorie 3: $\quad$ CHF 550

Kategorie 4: $\quad$ CHF 355

Kategorie 5: $\quad$ CHF 253

Kategorie 6: $\quad$ CHF 253

Kategorie 7: $\quad$ CHF 142

Kategorie 8: $\quad$ CHF O

Kategorie 9: $\quad \mathrm{CHF} O$

Kategorie 10: $\quad$ CHF O

Kategorie 11: $\quad$ CHF 178

Antrag ZV:

Genehmigung der Mitgliederbeiträge 2017

Beschluss:

Die Mitgliederbeiträge 2017 werden einstimmig genehmigt.

Die Delegierten stimmen nun über das konsolidierte Budget 2017 der FMH ab:

Antrag ZV:

Verabschiedung konsolidiertes Budget FMH 2017 Beschluss:

Das konsolidierte Budget FMH 2017 wird mit 150 Ja, 1 Nein und 2 Enthaltungen genehmigt.

\section{Budgetstabilisierungsmassnahmen}

\subsection{Reporting der Ergebnisse Q1 und Q2}

Anne-Geneviève Bütikofer/GS FMH erinnert an die von der ÄK am 28.10.2015 beschlossenen Massnahmen. Nach einem Evaluationsprozess im vergangenen Dezember wurde mit der Umsetzung der Massnahmen anfangs 201 rasch begonnen. Dank der grossen Anstrengungen sämtlicher Kader sowie aller involvierten Stellen und Organe konnten die Vorgaben für das 1. Semester zu 92\% zum erwarteten Resultat erreicht werden. Von den ursprünglich budgetierten Einsparungen von CHF 2,08 Mio. wurden im ersten Semester 2016 bereits CHF 1,9 Mio. umgesetzt. Die Differenz ist auf eine Verzögerung bei geplanten Massnahmen intern im Generalsekretariat sowie auf Zusatzausgaben bei den Organen zurückzuführen. Bereits heute steht jedoch fest, dass die von der ÄK am 30.10.2014 festgelegten Ziele, wie Erreichen eines finanziellen Gleichgewichts zwischen getätigten Ausgaben und Projekten sowie den generierten Einnahmen, die Konzentration auf 


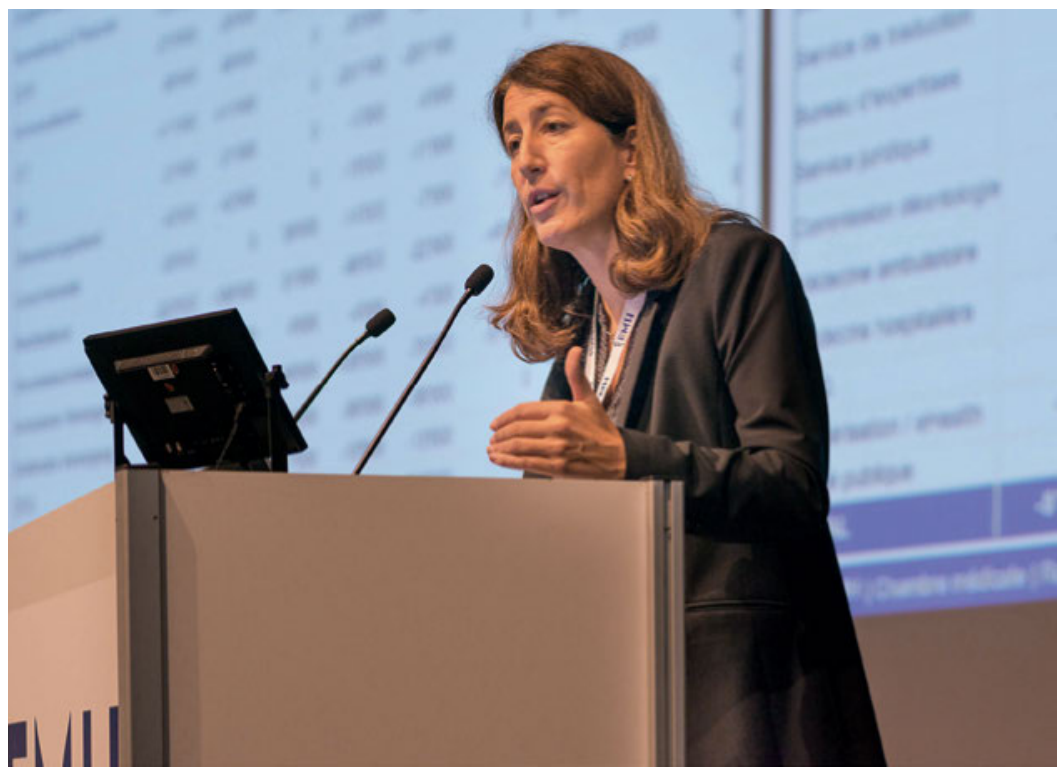

Die Generalsekretärin der FMH, Anne-Geneviève Bütikofer, berichtet über die Budgetstabilisierungsmassnahmen.

das Kerngeschäft und den Abbau von Nebenaufgaben erreicht und die angepeilten Einsparungen von $\mathrm{CHF}$ 4,06 Mio. realisiert werden können.

\subsection{Reduktion Sockelbeiträge EMH/SMSR/OMCT per 1.1.2018}

Jürg Schlup/Präsident FMH informiert einleitend über die von der GPK und der SMSR eingereichten Anträge, welche beim Generalsekretariat erst im Oktober 2016, also nach der DV vom 7.9.2016 eingegangen sind. Der ZV FMH hat bekanntlich nach der von der ÄK am 30.10.2014 beschlossenen Überprüfung der EMH dem Wirtschaftsprüfungsunternehmen KPMG den Auftrag für ein Audit erteilt. Die KPMG evaluierte für die EMH ein Verbesserungspotenzial von CHF 1,4 Mio. Daraufhin hat EMH dem ZV geeignete Massnahmen vorgeschlagen, um diese Verbesserung zu erreichen. Diese Vorschläge umfassen z.B. den Verzicht auf den Sockelbeitrag. Die Umsetzung dieser Massnahme hätte eine Reduktion des Sockelbeitrags per 1.1.2018 zur Folge. Der ZV FMH unterbreitete der DV am 7.9.2016 verschiedene Varianten. Variante 1 sah eine Teilreduktion von heute CHF 75 auf künftig CHF 45 für die Mitgliederkategorien 1, 2, 3, 5 und 6 vor. Variante 2 ging von einer schrittweisen Reduktion von heute CHF 75 auf CHF 0 aus. Die DV empfiehlt der $\ddot{\mathrm{AK}}$ Variante 1.

\subsubsection{Antrag GPK: Sockelbeitrag EMH/SMSR/ OMCT}

Adrian Sury/Präsident GPK erklärt, dass die GPK mit der Einreichung ihrer Anträge einen sinnvollen Mitteleinsatz bewirken will. Sie ist nicht gegen eine finanzielle
Unterstützung an die SMSR und den OMCT. Bevor jedoch über einen weiteren Sockelbeitrag entschieden werden soll, ist eine Prüfung der Finanzabschlüsse angezeigt. Ressourcen sollten nur dort eingesetzt werden, wo sie auch benötigt werden. Die EMH hat ihre Bücher bereits offengelegt und Verbesserungspotenziale aufgezeigt. Falls die Zeitschriften der SMSR und des OMCT ohne die Sockelbeiträge (die 2011 aus regionalpolitischen Gründen und zur Gleichbehandlung in Analogie zur EMH gesprochen wurden) effektiv finanziell in Bedrängnis geraten sollten, könnten im Falle eines verbleibenden strukturellen Defizits mit den beiden Gesellschaften separate Leistungsvereinbarungen abgeschlossen werden.

\subsubsection{Antrag SMSR: Ausgewogene Unterstützung der medizinischen Fachzeitschriften aus den Sprachregionen}

Pierre-Alain Schneider/Präsident SMSR weist darauf hin, dass sich die Schweizer Fachzeitschriften für Medizin nach der Fusion der beiden Zeitschriften Revue médicale de la Suisse Romande und Revue Médecine et Hygiène nach wie vor in einer schwierigen Phase befinden. Zwar hat der EMH-Verlag die Sparmassnahmen, wie Reduktion Sockelbeitrag, akzeptiert. EMH kann aber weiterhin auf die Unterstützung der FMH zählen, da die Publikation der SÄZ beim Verlag belassen wird. Die Ankündigung der EMH, wonach sie auf Subventionen verzichten und neu auch Dividenden ausschütten will, erstaunte die SMSR sehr. Sie bittet den ZV, unter Berücksichtigung der indirekten Vorteile, die dem EMH-Verlag gewährt werden, eine ausgewogene Unterstützung der Publikationen aus den drei Sprachregionen zu prüfen. Auf der Basis des entsprechenden Berichts soll die ÄK eine fundierte Entscheidung hinsichtlich des Sockelbeitrags EMH/SMSR/OMCT treffen. Der Antragsteller bittet die Delegierten, heute auf eine Entscheidung zu verzichten und erst nach Vorliegen der benötigten Informationen das weitere Vorgehen zu beschliessen.

Jürg Schlup/Präsident FMH eröffnet die Diskussion und legt nach den Voten der Delegierten das Abstimmungsprozedere fest. Als erstes wird über den Antrag Teil 2 des SMSR, anschliessend über den Antrag Teil 2 der GPK und als letztes über die Gegenüberstellung der Variante $1 \mathrm{ZV}$ und Variante 2 GPK abgestimmt.

Die GPK ist mit diesem Vorgehen einverstanden und hält auf die Frage von Jürg Schlup/ Präsident FMH fest, dass Teil 2 ihres Antrags den soeben festgelegten Abstimmungsablauf nicht blockiert. Teil 1 des Antrags der GPK bezieht sich auf den Sockelbeitrag, Teil 2 auf die Prüfung der Finanzabschlüsse von SMSR und OMCT. 
Abstimmung 1: Teil 2 des Antrags SMSR:

Antrag SMSR, Teil 2:

Die ÄK prüft die Situation für 2018 erneut, sobald der ZV einen Bericht über die ausgewogene Unterstützung der medizinischen Fachzeitschriften aus den drei Sprachregionen vorgelegt hat. Dabei sind insbesondere der Wettbewerbsvorteil, den der EMH-Verlag durch die Publikation der SÄZ und den gemeinsamen Versand des Schweizerischen Medizin-Forums an alle FMH-Mitglieder erhält, sowie das Einsparpotenzial im Falle einer Publikation der SÄZ durch einen anderen Verlag zu erwägen.

Beschluss:

Der Antrag wird mit 74 Nein, 66 Ja und 6 Enthaltungen abgelehnt.

Abstimmung 2: Teil 2 des Antrags GPK:

Antrag GPK, Teil 2:

Hinsichtlich SMSR und OMCT: Prüfung der Finanzabschlüsse durch ein externes Revisionsbüro, Ergebnisverbesserungspotenzial. Im Fall eines verbleibenden strukturellen Defizits werden separate Leistungsverträge mit den beiden Gesellschaften abgeschlossen, die jährlich überprüft werden müssen. Deren Höhe wird mit einem verbleibenden Sockelbeitrag der Mitglieder finanziert, der durch einen Ärztekammerbeschluss im Mai 2017 festgelegt wird.

Beschluss:

Der Antrag wird mit 116 Ja, 13 Nein und 16 Enthaltungen angenommen.

Vor der dritten Abstimmung, bei welcher sich die Delegierten entweder für Variante 1 des ZV oder für Variante 2 der GPK entscheiden können, sind sich die Anwesenden über die Konsequenzen dieser Varianten uneinig. Einige Delegierte wünschen einen Rückzug der Variante 2 der GPK. Jürg Schlup/Präsident FMH fragt die GPK, ob sie mit einem Rückzug einverstanden sei. Adrian Sury/Präsident GPK hält am Antrag fest und erklärt noch einmal, dass dieser das weitere Prozedere nicht blockiere. Die GPK lehnt den Rückzug ihres Antrags ab.

Jürg Schlup/Präsident FMH stellt folgenden Ordnungsantrag:

Ordnungsantrag Schlup:

Die Delegierten sind mit der Gegenüberstellung der Variante $1 \mathrm{ZV}$ - empfohlen von ZV und DV versus Variante 2 der GPK - empfohlen von der GPK - einverstanden.

Beschluss:

Der Ordnungsantrag wurde mit $117 \mathrm{Ja}, 1$ Nein und 15 Enthaltungen angenommen.

Bei der letzten Abstimmung geht es um die Festlegung des Sockelbetrags für 2018 und Folgejahre. Variante 1 des ZV schlägt eine Teilreduktion von heute CHF 75 auf künftig CHF 45 für die Mitgliederkategorien 1, 2, 3, 5 und 6 vor. Die DV empfiehlt der ÄK diese Variante. Variante 2 der GPK will eine 50\% Reduktion von heute CHF 75 ab 2018 und Aufhebung ab 2019. Sollte der verlangte Bericht $\mathrm{zu}$ anderen Schlussfolgerungen kommen, muss die ÄK den Sockelbeitrag für 2019 neu beschliessen.

Abstimmung 3: Gegenüberstellung Variante 1 des ZV versus Variante $2 \mathrm{GPK}$

Antrag:

Gegenüberstellung Variante 1 des ZV versus Variante 2 der GPK

Beschluss:

Für die Variante 1 des ZV stimmen 14, für die Variante 2 der GPK 127 Delegierte. 8 Delegierte enthalten sich der Stimme. Variante 2 der GPK ist somit angenommen.

\subsection{Erhöhung Mitgliederbeitrag für Kategorie 5}

Ein weiterer Vorschlag der EMH, mit welchem sie ein Verbesserungspotenzial realisieren will, ist die Übernahme der Portokosten durch die FMH für Auslandabonnemente. Diese Versandkosten für die Schweizerische Ärztezeitung und das Swiss Medical Forum sind von den Auslandabonnenten per 1.1.2018 zu übernehmen und haben eine Erhöhung des Mitgliederbeitrags für die Kategorie 5 zur Folge.

Antrag:

Entlastung von EMH AG durch Streichung der Portokosten für Auslandabonnementen und Übernahme dieser Kosten durch die Ärztinnen und Ärzte mit Wohnsitz und Berufstätigkeit im Ausland (Kategorie 5)

Erhöhung des Mitgliederbeitrags allein für die Kategorie 5 von heute $1 / 4$ auf neu $2 / 5$ von heute CHF 178 auf neu CHF 284.

Diese Beitragserhöhung tritt in demjenigen Jahr in Kraft, in welchem der Sockelbeitrag erstmals reduziert wird, nämlich ab 1.1.2018.

Beschluss:

Der Antrag wird mit 112 Ja, 9 Nein und 11 Enthaltungen angenommen.

\section{Wahlen, Bestätigungswahlen}

6.1 Bestätigungswahl von nominierten DV-Delegierten

Die FMH-Statuten sehen vor, dass die Delegierten und Ersatzdelegierten der DV durch die ÄK bestätigt werden. Die zu bestätigenden Delegierten sind aus- 
schliesslich stimm- oder antragsberechtigte ÄK-Delegierte (FMH-Statuten Art. 36).

\section{Antrag:}

Die antragsstellenden Organisationen VEDAG, SFSM, SMSR und fmCh beantragen der ÄK, die folgenden Delegierten neu für die Delegiertenversammlung der FMH zu bestätigen:

\section{VEDAG}

Bisher: Marcel STAMPFLI, Biel

Neu ab 27.10.2016: Florian LEUPOLD, Breitenbach SFSM

Bisher: Jürg NADIG, Bülach

Neu ab 27.10.2016: Emanuel CHRIST, Niederscherli SMSR

Bisher: Véronique MONNIER-CORNUZ, Lausanne Neu ab 27.10.2016 : Philippe EGGIMANN, Sullens fmCh

Bisher: Urban LAFFER, Präsident

Neu ab 27.10.2016: Thomas EGGIMANN, Kreuzligen

Bisher: Daniel ACKERMANN, Scuol

Neu ab 27.10.2016: Gianfranco MATARELLI, Liestal

Beschluss:

Die Delegierten werden mit 150 Ja und 2 Enthaltungen bestätigt.

\subsection{Bestätigungswahl von nominierten DV-Er- satzdelegierten}

Die FMH-Statuten sehen vor, dass die Delegierten sowie Ersatzdelegierten der DV durch die ÄK bestätigt werden. Die zu bestätigenden Delegierten sind ausschliesslich stimm- oder antragsberechtigte ÄK-Delegierte (FMH-Statuten Art. 36a).

\section{Antrag:}

Die antragsstellende Organisationen VSAO und SFSM beantragen der ÄK, die folgenden Ersatzdelegierten neu für die Delegiertenversammlung der FMH zu bestätigen:

VSAO:

Bisher: Zweiter Ersatzdelegierter vakant

Neu ab 27.10.2016: Gert Printzen, Luzern

SFSM

Bisher: Emanuel Christ, Niederscherli

Neu ab 27.10.2016: Stefan Greuter, Sargans

Beschluss:

Die Ersatzdelegierten werden mit 150 Ja, 2 Nein und 10 Enthaltungen bestätigt.

\section{Gastreferat}

Jürg Schlup/Präsident FMH begrüsst Prof. Dr. Samia Hurst als Gastreferentin. Die Direktorin des Instituts für Ethique Histoire Humanités der Universität Genf

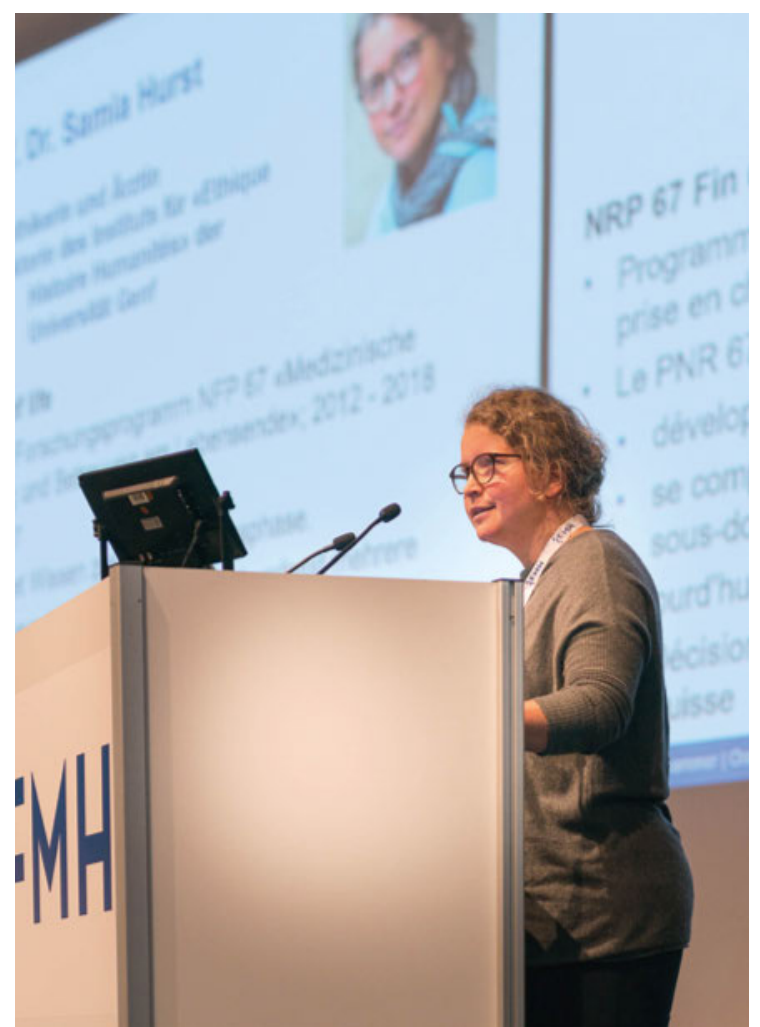

Prof. Dr. Samia Hurst, Medizinerin und Ethikerin, stellt ein Teilprojekt aus dem Nationalen Forschungsprogramm "Lebensende» (NFP 67) vor.

hat im Nationalen Forschungsprogramm 67 (NFP 67) «Lebensende» mitgeforscht. Das NFP 67 «Lebensende» will neue Erkenntnisse über die letzte Lebensphase des Menschen jeden Alters gewinnen. In 33 Forschungsprojekten werden Handlungs- und Orientierungswissen für Entscheidungsträgerinnen und -träger im Gesundheitswesen und in der Politik sowie für die betroffenen Berufsgruppen erarbeitet. So individuell wie die Menschen leben, so individuell sterben sie. Doch unter welchen Bedingungen sterben sie und welche Entscheidungen werden am Lebensende getroffen? Prof. Hurst referiert zum Modul NFP 67 «Entscheidungen am Lebensende: Häufigkeit und Tendenz in der Schweiz».

\section{Qualitäts-Charta}

Die ärztliche Tätigkeit ist von der Verantwortung gegenüber Patientinnen und Patienten geprägt. Die Qualität der eigenen Leistungen gehört als selbstverständlicher Teil zur Arbeit und ist zum Nutzen für Patienten, Angehörige und Ärzte. Qualitätsarbeit ist in allen Aspekten zu gewährleisten und zu fördern. Um diese Aktivität bezüglich Qualitätsarbeit zu bündeln und auszuweisen, wurde die Schweizerische Akademie für Qualität und Medizin (SAQM) 2012 gegründet. 


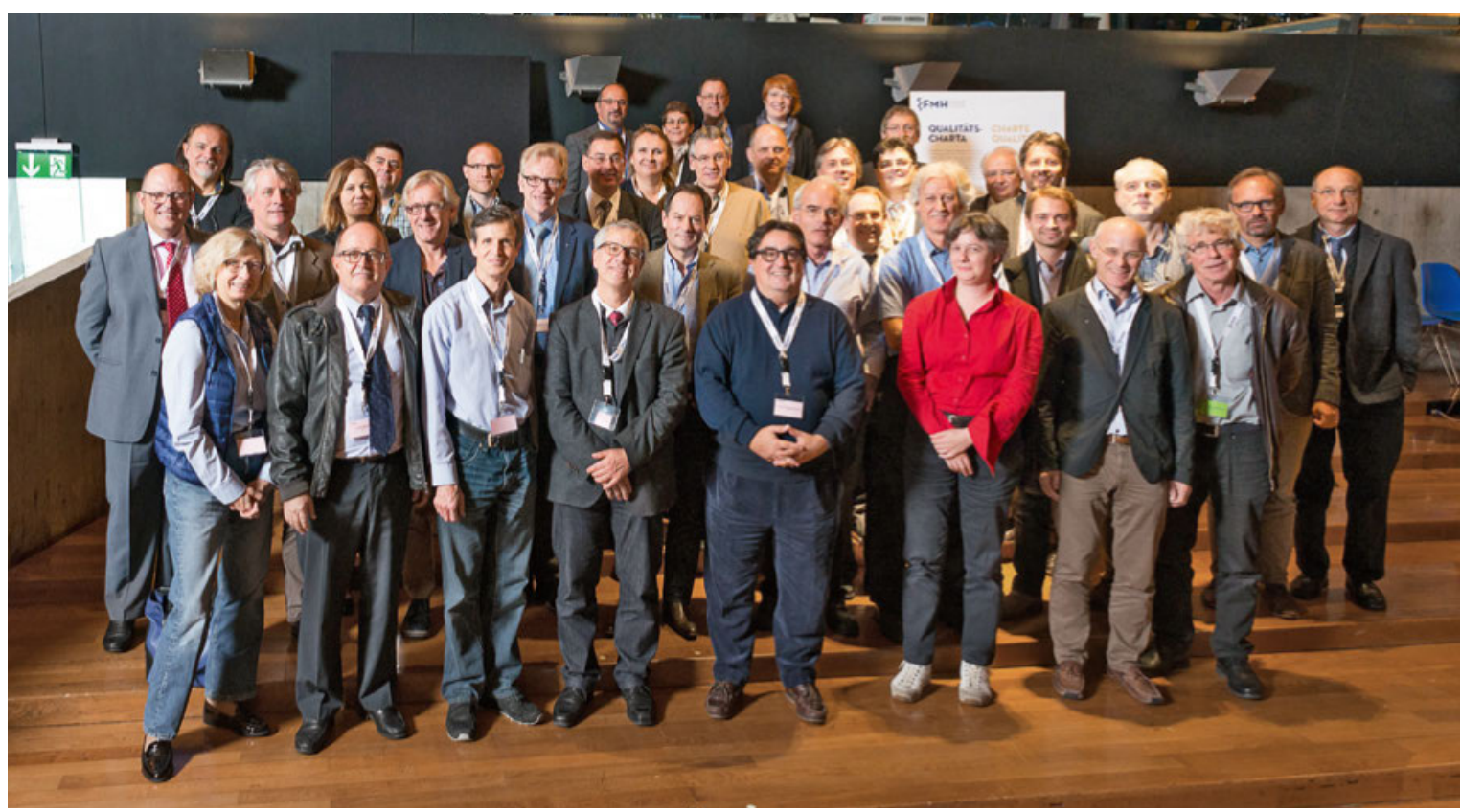

58 Ärzteorganisationen unterzeichneten die Qualitäts-Charta und bekennen sich damit zu Transparenz, Verbindlichkeit und Nachhaltigkeit in der Qualität.

Christoph Bosshard/ZV FMH informiert über Ziel und Inhalt der Qualitäts-Charta, welche in den vergangenen zwei Jahren von der SAQM erarbeitet wurde. Die Charta hat zum Ziel, die Qualitätsarbeit in der Medi-zin verbindlich und transparent zu gestalten. Nach einer breit abgestützten Vernehmlassung innerhalb der Ärzteschaft ist die Qualitäts-Charta finalisiert. Die unterzeichnenden Ärzteorganisationen bekennen sich zu Qualitätsgrundprinzipien in den Bereichen Transparenz, Verbindlichkeit, Nachhaltigkeit, Korrektheit, Kompetenzen und auch Ressourcen. Gemeinsam stellen sie auch Forderungen gegenüber der Politik, Verwaltung und weiteren Gesundheitsorganisationen. Die symbolische Erst-Unterzeichnung erfolgte anlässlich der heutigen ÄK. Die Unterzeichnung ist und bleibt freiwillig und ist auch weiterhin möglich.

\section{Strategie FMH 2017-2020}

Jürg Schlup/Präsident FMH erläutert die vom ZV erarbeitete Strategie der FMH für die neue Legislatur. Die Herausforderungen wurden mittels dreier Befragungen eruiert. Schriftlich befragt wurden die Delegierten der ÄK, 12 Gesundheitsexpertinnen aus Wirtschaft und Politik sowie die Kader des Generalsekretariats FMH. Bei der Formulierung der strategischen Ziele wurde auf eine sinnvolle Kontinuität der Ziele aus der letzten Legislatur geachtet. Die Delegierten, Experten und Kader nennen hochrelevante Herausforderungen sowohl für die Ärzteschaft wie auch für die Gesund- heitsversorgung. Mit deutlichem Abstand am häufigsten wurden Tarifthemen angesprochen, d.h. die Tarifrevision und der Erhalt der Tarifautonomie. Weiter von Wichtigkeit sind der Zusammenhalt des Verbandes, die wirksame Interessenvertretung, eHealth, Qualität, Zulassungssteuerung, die Gestaltung und Attraktivität der Berufsbildung in der Zukunft.

Aus diesen Herausforderungen hat der ZV folgende Hauptziele definiert:

- Proaktive Gesundheitspolitik mit einer Stimme

- Qualität weiterentwickeln und sichtbar machen

- Entwicklung der Berufsidentität und -ausübung

- Tarifautonomie und sachgerechte Entschädigung in der ambulanten Versorgung

- Tarifgestaltung und sachgerechte Entschädigung in der stationären Versorgung

- Digitale Transformation in der Medizin im Dienste der Ärzteschaft, Patientinnen und Patienten

- Public Health unterstützen und fördern

- Professionelle Supportleistungen des GS gewährleisten

Wie bereits seit 2013 praktiziert, werden die strategischen Ziele und die im Rahmen der Umsetzung definierten Massnahmen einmal pro Jahr durch den ZV überprüft und bei Bedarf ergänzt.

Aufgrund des fehlenden Quorums findet keine Abstimmung statt. Bei der durchgeführten Konsultativabstimmung werden die Ziele für die Legislatur von 63 Delegierten mit 1 Enthaltung und ohne Gegenstimme unterstützt. Die strategischen Ziele für die 
Legislaturperiode 2017-2020 werden der ÄK im Mai 2017 unterbreitet.

\section{Information MARS}

Aufgrund der Aktualität und Bedeutung seitens FMH wird dieses Traktandum ausführlicher als das übrige Beschlussprotokoll geführt.

Christoph Bosshard/ZV FMH informiert über den aktuellen Projektstand. Die Pflicht zur Datenlieferung seitens der Leistungserbringer war ursprünglich im seit 2009 geltenden Art. 22a KVG definiert und wurde ab 1.1.2016 inhaltsgleich zum Art. 59a. Die entsprechende Anpassung in der Verordnung über die Krankenversicherung (KVV) zur Umsetzung von Art. 59a trat am 1.8.2016 in Kraft. Art. 30c dieser Verordnung bezieht sich auf ein Datenbearbeitungsreglement, welches nun zur Anhörung zugestellt wurde. In Umsetzung dieser Gesetzesbestimmung erhielt das BFS den Auftrag, das als MARS bezeichnete Informationssystem aufzubauen und zu betreiben. Teilprojekt 3 sieht eine jährliche Datenlieferung von Arztpraxen und ambulante Zentren vor. Die Auswertungen aus dem Testbetrieb (Piloterhebungen 2015 und 2016) waren erfolgreich, weshalb das BFS die Erhebung der Daten von Arztpraxen und ambulanten Zentren mit Referenzjahr 2015 am 15.11.2016 startet. Die Daten dieser ersten Erhebung werden nur für statistische und nicht für auf- sichtsrechtliche Zwecke verwendet, da das Datenbearbeitungsreglement gemäss Art. 30c KVV noch nicht in Kraft ist. Zentral für die in einem Jahr folgende Erhebung, welche dann auch für aufsichtsrechtliche Zwecke gemäss KVG genutzt wird, ist eine für die Ärzteschaft transparent und gesetzeskonform geregelte Datenerhebung, -weitergabe und -verwendung. Die Datenweitergabe und -verwendung muss im Datenbearbeitungsreglement klar definiert und ausgeführt sein. Alle Mitglieder haben nun Gelegenheit, innerhalb der verlängerten Anhörungsfrist bis Ende Januar 2017 ihre Stellungnahme einzureichen.

Vor allem dieses sich noch in Anhörung befindende Datenbearbeitungsreglement führte innerhalb der Ärzteschaft zu Unsicherheiten und Fragen. Das hat die FMH-Führung bewogen, die Verantwortlichen des Bundesamts für Statistik (BFS), des Bundesamts für Gesundheit (BAG) und des Eidgenössischen Datenschutz- und Öffentlichkeitsbeauftragten (EDÖB) sowie den diesbezüglichen juristischen Berater einzuladen. Der Präsident begrüsst Adrian Lobsiger/Eidg. Datenschutz- und Öffentlichkeitsbeauftragter (EDÖB), Oliver Peters/Vizedirektor und Leiter Direktionsbereich Kranken- und Unfallversicherung (BAG), Marco D’Angelo/ Abteilungschef Gesundheit und Soziales (BFS), Jacques Huguenin/Sektionschef Gesundheitsversorgung (BFS), Sabine Trolliet/Juristin (EDÖB) sowie RA Michel Meier.

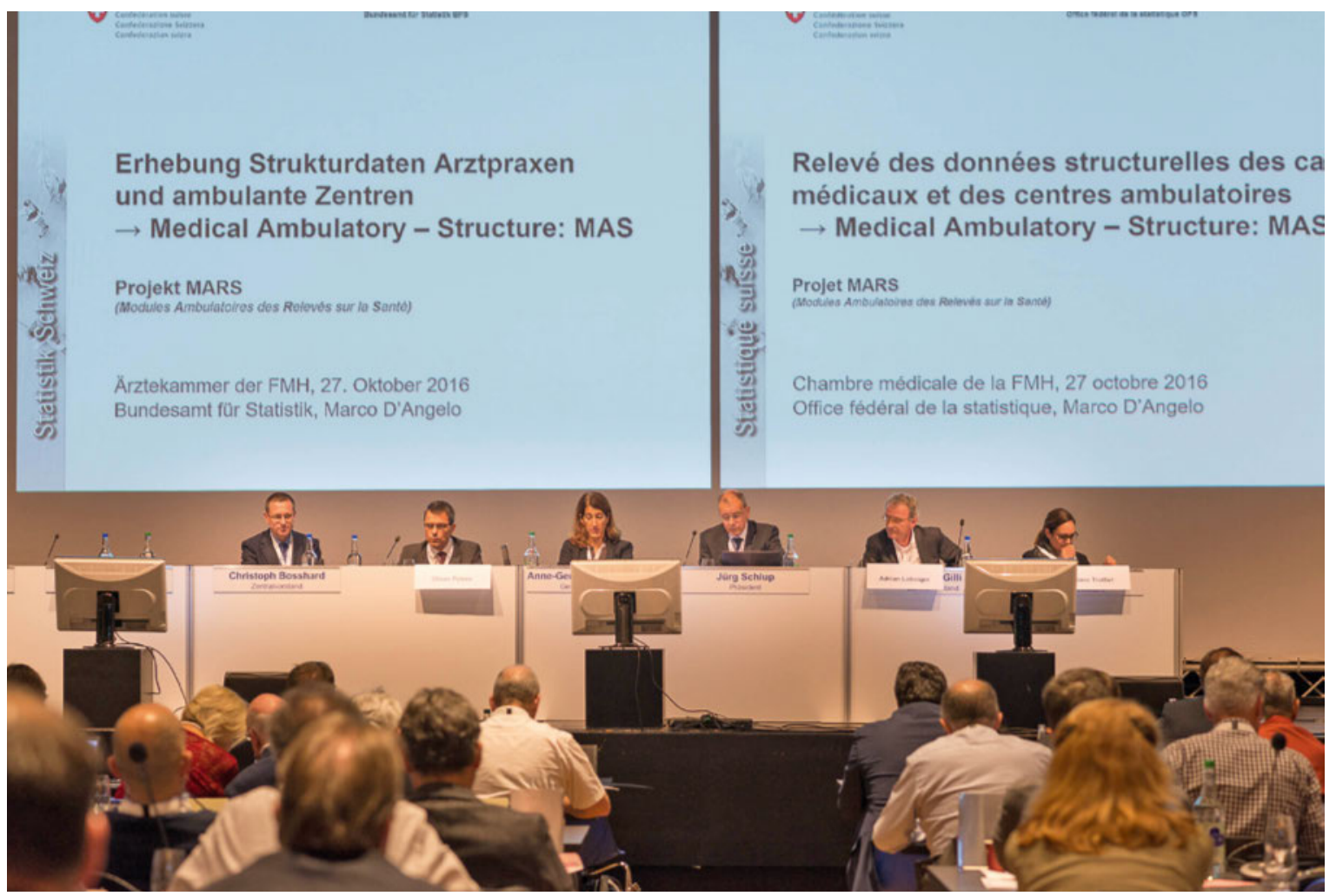

Vertreter der Bundesverwaltung und der EDÖP stellen sich den Fragen der Delegierten zum Projekt MARS. 
Marco D'Angelo/Abteilungschef Gesundheit und Soziales $B F S$ stellt den Zweck und den Ablauf der Datenerhebung zu den Arztpraxen und ambulanten Zentren (MAS) vor. Der Erhebungsstart ist nach wie vor für den 15.11.2016 geplant. Die Anhörungsfrist für das Bearbeitungsreglement wurde inzwischen auf den 31.1.2017 verlängert. Wo im stationären Bereich bereits eine gute statistische Datengrundlage existiert, fehlt eine solche im ambulanten Bereich. Entsprechend der parlamentarischen Vorstösse, die schliesslich zum Projekt MARS führten, wird diese Lücke nun geschlossen. Die nun erstmals zu erhebenden Daten werden im ersten Jahr ausschliesslich zu statistischen Zwecken und nicht zu Aufsichtszwecken gemäss Art. 59a KVG erhoben und verwendet. Es werden sowohl Struktur- wie auch Betriebs- und Finanzdaten erhoben. Der Erhebungsinhalt besteht aus drei Bereichen. Im ersten geht es um die Informationen zum Standort (Angaben zum Standort/Praxis, Infrastruktur und Ausstattung, Ausund Weiterbildungsangebot, Anzahl Patienten /Patientenkontakte). Der zweite Bereich betrifft die Personen (Ärzte/Ärztinnen anonymisiert, Nicht ärztliche Berufsgruppen (aggregiert)) und der dritte die Finanzen (Aufwand, Ertrag KVG / Gesamt, Betriebsergebnis).

Nach dem zweiten Testbefragungspilot konnte der Zeitaufwand für den Fragebogen von 2 auf 1,5 Stunden reduziert werden. Die drei Schnittstellen zur Datenübernahme von Datensammlungen der Ärzteschaft (FMH, NewIndex, RoKo) und zum BAG (Medreg) haben sich bewährt. Die Erhebung verfolgt zwei Zwecke: die statistische Verwendung nach Bundesstatistikgesetz (BStatG) und die aufsichtsrechtliche Verwendung nach Art. 59a KVG inkl. der entsprechenden Artikel der KVV. Hinsichtlich der Datenweitergabe gilt die Unterscheidung zwischen BStatG und KVG. Nach BStatG werden die Daten der juristischen und natürlichen Personen anonymisiert. In der angepassten Verordnung der KVV, welche am 1.8.2016 in Kraft trat, sieht Art. 30c ein Bearbeitungsreglement vor. Dieses Bearbeitungsreglement ist zur Zeit in Anhörung und wird voraussichtlich erst im Frühjahr 2017 rechtskräftig.

Jürg Schlup/Präsident FMH dankt dem Referenten für seine Ausführungen und eröffnet die Diskussion.

FMH: Ist der 90-minütige Aufwand für den Fragebogen pro Monat/Woche oder Jahr berechnet?

BFS: 90 Minuten pro Jahr. Dieser Wert entspricht der Auswertung des Testbefragungspilots.

FMH: Hat das BFS Zugriff auf die Daten von Ärzten, die an einem Trust-Center angeschlossen sind und ist der Fragebogen damit zu 100 Prozent ausgefüllt? BFS: Durch die Möglichkeit des Einbezugs der bestehenden Datensammlungen wird die Erfassung der Daten im Fragebogen vereinfacht. Diese müssen nicht noch einmal eingegeben werden. Mit der Authentifizierung und dem Einverständnis des einzelnen Leistungserbringers können die Daten verwendet werden. FMH: Gibt es bei der ersten Datenerhebung, die statistischen Zwecken dienen soll, noch weitere Zwecke, die verfolgt werden?

BAG: In der Verordnung, die am 1.8.2016 in Kraft gesetzt wurde, ist festgelegt, dass die Datensammlung auch statistischen Zwecken dient. Das Parlament hat aber bei der Verabschiedung des KVG gewollt, dass die gleichen Daten nicht ein zweites Mal für Zwecke der Qualität (Qualitätsindikatoren, Wirtschaftlichkeit der Leistungen) erhoben werden müssen. Das Gesetz regelt, dass das BFS ebenfalls die notwendigen Daten erhebt, die nachher zu Qualitätszwecken verwendet werden sollen. Die medizinische Leistungserbringung findet vermehrt ambulant statt. Aus diesem Grunde ist es für Qualitätszwecke wichtig, den Zusammenhang zwischen ambulanten und stationären Behandlungen herstellen zu können. Das BAG ist aus Gründen der kleinen und sehr variablen Patientenkollektive nicht daran interessiert, Einzelpraxenauswertungen zur Qualität zu machen. Es geht mehr darum, regionale Versorgungsunterschiede festzustellen, um daraus die notwendigen Lehren für die Weiterentwicklung des Gesundheitswesens zu ziehen.

FMH: Die Ärzteschaft gibt bereits ihre Daten in RoKo ein. Weitere zusätzliche Statistiken sind ein Mehraufwand sowohl für die Bundesstellen wie für die Ärzte. Was ist das jährliche Budget der Verwaltung?

BFS: Das BFS hat für die Erfüllung dieses gesetzlichen Auftrags die entsprechenden finanziellen Mittel im Rahmen ihres Budgets beantragt.

BAG: Die Erfassung und Publikation von Qualitätsindikatoren ist wichtig, um die medizinischen Leistungen zu verbessern. Das Parlament hat mit Nachdruck die Einführung von Qualitätskriterien und Indikatoren im ambulanten Bereich gefordert. Das BAG kennt z.B. den Prozentsatz von Operationen im schweizerischen ambulanten Bereich nicht. In anderen Ländern kennt man diesen Prozentsatz genau, z.B. wie viele Hüftprothesen, Knieprothesen und andere Operationen im stationären oder ambulanten Bereich vorgenommen werden. Hier ist die Schweiz im Vergleich zu anderen Ländern im Verzug und es muss alles unternommen werden, das gleiche Niveau zu erreichen.

FMH: Wie, wann und in welcher Art und Weise erhalten die Ärzte die Daten zurück?

BFS: Die Daten, die nach BStatG erhoben und zur Verfügung gestellt werden, sind auch für die Öffentlichkeit und werden im internationalen Kontext anonymisiert verwendet. Die Daten, die nicht anonymisiert sind, werden gemäss den Zweckbestimmungen des KVG an 


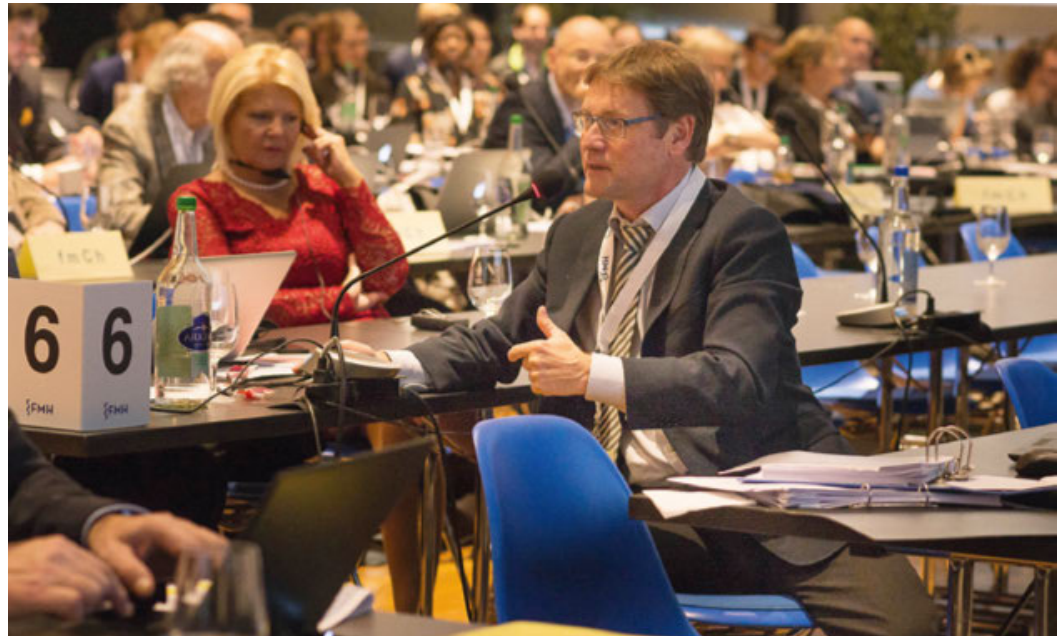

Hanspeter Kuhn, Leiter der Abteilung Rechtsdienst der FMH, beantwortet eine Frage.

das BAG weitergeliefert. Das BFS kann Daten, die nicht anonymisiert sind, nicht an Dritte weitergeben, ausser es wird dazu bevollmächtigt. Die Daten können nur mit einem gesetzlichen Auftrag weitergegeben werden. Bei H+ ist es so, dass das BFS dazu eine entsprechende Vollmacht erhält.

EDÖB: Die Auswertung durch das BFS muss in einem Verhältnis zum Zweck stehen. Letztlich geht es hier auch um Kosteneffizienz und Qualität. Das Endresultat darf keine Rückschlüsse auf Patienten oder Datenlieferanten ermöglichen, soweit dies nicht für die Zweckerreichung unbedingt notwendig ist.

FMH: Gemäss Präsentation ist eine Datenerhebung auch bei den Apothekern und bei der Spitex geplant. Es ist schwierig, die Qualität der Arztpraxen zu verbessern, solange nicht alle Parameter, auch der sozial-demographischen Situation der Patienten bekannt sind. Wann startet die Statistik zu den Apothekern? Der Bundesrat hat letzthin entschieden, diesen mehr Kompetenzen für chronische Kranke und Notfälle zu geben. Es ist nicht nachvollziehbar, weshalb die Ärzteschaft zu einer Erhebung gezwungen wird, die Apotheker jedoch nicht.

BAG: Im Moment ist nicht vorgesehen, dass die Apotheker medizinische Leistungen erbringen, die den $\mathrm{Pa}$ tienten in Rechnung gestellt werden. Es handelt sich hier um ein Pilotprojekt im Rahmen der Polymedikation, für welches von den Apothekern und ihrem Verband Angaben verlangt werden. Bei der Spitex ist ebenfalls ein Projekt geplant. Es geht nicht um die Erfassung und Überprüfung der Qualität im Einzelnen, sondern um den Erhalt einer Gesamtübersicht über die Langzeitpflege zuhause und in spezialisierten Institutionen. Für die Arztpraxen ist es noch schwieriger als für die Spitäler oder gar unmöglich, da das Kollektiv der Patienten kleiner und die Komplexität grösser ist. Das
BAG interessiert eine Übersicht der regionalen Versorgung und der verschiedenen Gewohnheiten und der anschliessende Vergleich zwischen den ambulanten und eventuell später folgenden stationären Behandlungen. Heute werden bereits viele onkologische Behandlungen im ambulanten Bereich ausgeführt und Operationen in den ambulanten Bereich verschoben. Es wird vom BAG keine nominale Publikationen pro Arztpraxis geben, sondern eine nach Krankheitsart, nach Regionen usw.

FMH: Was ist unter einer qualitativen Einschätzung zu verstehen und wie soll die Qualität der Leistungen statistisch gemessen werden?

BAG: Auf internationalem Niveau gibt es bereits Studien, wie ein Spitalaufenthalt durch eine gute ambulante Betreuung der chronisch Kranken vermieden werden kann. Es ist interessant, den Zusammenhang zu erstellen zwischen den Studien, welche auf Basis von Spitalstatistiken und medizinischen Statistiken, welche seit 15 Jahren bestehen und keine Probleme mit dem Datenschutz mit sich brachten, sowie den Resultaten von MARS. Die andere Möglichkeit ist die Evaluation der erbrachten oder nicht erbrachten Leistungen für einen Patiententyp, der unter einer gewissen Krankheit leidet, um zu erkennen, wie die regionalen Standards und Unterschiede sind. Die Studien, die in gewissen Regionen in der Schweiz durchgeführt wurden, haben gezeigt, dass die Anwendung von anerkannten Guidelines sehr unterschiedlich ist. Diese Erkenntnis ist sowohl aus Sicht der öffentlichen Gesundheit wie auch aus Sicht der Optimierung der Behandlungen interessant.

FMH: Um das zu tun, braucht das BAG keine zusätzlichen Daten. Weshalb sollen die Ärzte noch weitere Daten liefern, die weder anonymisiert noch aggregiert sind?

EDÖB: Die Daten müssen zwingend anonymisiert sein und sollen keinen Rückschluss auf Personen geben. Eine Re-Identifizierung muss ausgeschlossen sein. Die Aufgabe des EDÖB ist es, zu kontrollieren, dass dieses Risiko ausgeschlossen wird.

FMH: Werden hier nicht die Wirtschaftlichkeitsprüfung der medizinisch erbrachten Leistungen einerseits und die Wirtschaftlichkeit der Praxisführung andererseits vermischt? Das sind zwei verschiedene Fragen. Wenn von Qualität gesprochen wird, muss klar sein, ob über die Qualität einer Praxisführung gesprochen wird und ob ein Mandat auf gesetzlicher Ebene für die Kontrolle der Qualität der Leistungserbringung besteht.

BAG: Bereits heute weiss man, dass ein Patient in der Schweiz durchschnittlich viermal im Jahr zum Arzt geht und die Konsultation durchschnittlich CHF 200 kostet. Was man aber nicht kennt, sind die Überlebens- 
chancen eines Patienten, der eine onkologische Krankheit hat, je nachdem wo er in einer bestimmten Region des Landes behandelt worden ist oder je nach Onkologen, den er besucht oder nach Behandlung, die er erhalten hat. Diese Daten gibt es noch nicht. Viele medizinische Aktivitäten werden im ambulanten Bereich durchgeführt. Das Interesse, mehr über positive oder negative Resultate von gewissen Behandlungen und Krankheitsarten zu erhalten, wird aber immer wichtiger. Die Wirtschaftlichkeit der Praxisführung ist Sache des einzelnen Arztes oder der Versicherer und interessiert das BAG nicht. Das BAG interessiert die Wirtschaftlichkeit der Leistungen, welche den Krankenversicherer auf nationalem Niveau in Rechnung gestellt werden. Die Daten der Einzelpraxen sind den Versicherern schon bekannt, das BAG muss sich nicht noch zusätzlich darum bemühen. Wie bereits erwähnt, das BAG interessiert die Leistungen, die klinischen Resultate und deren Kosten. Dafür genügen leider die bereits erwähnten Angaben, z.B. wie oft ein Versicherter zum Arzt geht, nicht. FMH: Geht es bei der Erhebung um die Daten 2015 oder 2016? Die Datenerhebung zu den Erkrankungen, Diagnosen usw. würde ja bedeuten, dass ebenfalls die Diagnostikcodes hinterlegt werden.

BFS: Das BFS erstellt und betreibt die Infrastruktur für die Datenerhebung. Die Frage der Diagnose, die sich im Projekt mit den Spitälern mit den ambulanten Patientendaten auch gestellt hat, ist noch nicht geklärt. Es ist Gegenstand der laufenden Arbeiten, die Diskussionen betreffend dieser Thematik weiterzubringen. Dies kann jedoch noch andauern.

FMH: Das Bearbeitungsreglement ist noch nicht anwendungsbereit und in Kraft. Mit der Datenerhebung beginnt man jedoch vor der Inkraftsetzung. Ist das korrekt?

BFS: Die Erhebung wird ausschliesslich auf der Basis des BStatG vorgenommen. Das Bearbeitungsreglement ist bis Ende Januar 2017 in Anhörung. Die Anhörungsresultate werden genutzt, um das Reglement zu finalisieren und danach in Kraft zu setzen. Es geht hier um eine erste Vollerfassung, weshalb diese eine spezielle Behandlung erfährt.

BAG: Die Daten dieser Erhebung werden nur dem BFS übermittelt und von diesem verwendet. Da das Bearbeitungsreglement, welches den aufsichtsrechtlichen Gebrauch klar regeln soll, noch nicht genehmigt ist, ist die Weitergabe der Daten an die Kantone, an das BAG, an die Versicherer usw. für die diesjährig startende Erhebung blockiert.

FMH: Alle Organisationen, welche Qualitätskontrollen durchführen, haben dafür bezahltes Personal. Werden die Ärzte diese Aufgabe ohne Bezahlungen vornehmen müssen?
BAG: Die heute gültige Tarifstruktur sieht Möglichkeiten vor, Dokumentationsarbeiten zu verrechnen. FMH: Sowohl die Vertreter des BFS wie auch der Vertreter des BAG haben versichert, dass die Daten nur für statistische Zwecke verwendet werden und beim BFS verbleiben. Wer garantiert, dass diese Zahlen nicht rückwirkend noch vom BAG beim BFS angefordert werden?

BAG: Das BAG kann garantieren, dass sie diese Zahlen nicht gebrauchen. Das Interesse liegt in der Entwicklung von Qualitätsindikatoren und dem Erhalt von Informationen, wie sich das Leistungsvolumen im Verhältnis zum Leistungsinhalt entwickelt. Deshalb braucht es Zahlen von genügender Qualität wie auch Bearbeitungsreglemente, die für die Ärzteschaft, den Datenschutzbeauftragten und das BAG stimmen. Das BAG ist vor allem an den Leistungen interessiert und weniger an irgendwelchen namentlichen Angaben über einzelne Personen.

BFS: Das BFS wird die Daten nicht nachträglich liefern. Das würde dem widersprechen, was bis jetzt getan wurde.

EDÖB: Das wäre ein Zweckänderung und Zweckänderungen sind grundsätzlich unzulässig.

FMH: Als dritter Empfänger der Statistiken sind gemäss den Angaben des BFS die Krankenversicherer aufgeführt. Diese kennen jedoch bereits die Ärzte individuell durch die Prüfung der Wirtschaftlichkeit, Wirksamkeit und Zweckmässigkeit der Behandlungen. Haben diese mit ihrem Wissen nicht einen grossen Vorsprung oder Vorteil gegenüber dem Staat und den Kantonen?

BAG: Die Daten werden an die Versicherer nur in aggregierter Form übermittelt. Die Versicherer verfügen heute schon über detaillierte Abrechnungsdaten der Versicherten und der Leistungserbringen. Sie benötigten hierzu den Bund nicht.

FMH: Es ist löblich, die Qualität der Leistungen zu verbessern. Die Forderung nach ständig mehr Statistiken bedeutet für die Ärzte einen hohen administrativen Aufwand. Die Lieferung der vom BAG gewünschten Angaben erfordert einen grösseren Aufwand als 90 Minuten pro Jahr. Es sollte ein Informatiksystem evaluiert werden, welches die bereits erfassten Angaben schon integriert hat.

BAG: Ein einziges System, wo alle Daten nur einmal eingegeben und anschliessend auf verschiedene Weise genutzt werden können, wäre ideal. Leider ist dies sehr komplex. Es ist wie beim Ausfüllen der Steuererklärung. Beim ersten Mal dauert es viel länger. Ein Problem besteht auch dort, wo Diagnostikcodes eingegeben werden müssen. Dies kommt bestimmt einem kulturellen, technischen und administrativen Wandel 
gleich, dem man die nötige Zeit und Flexibilität geben muss. Heute werden bereits verschiedene Kodierungssysteme genutzt. Im stationären Bereich ist die Erfassung einfacher, da in den Spitälern die Diagnostikcodes im System bereits hinterlegt sind. Im ambulanten Bereich wäre dies zeitintensiver und wird noch viele Diskussionen zwischen der FMH, dem BAG und dem BFS mit sich bringen.

FMH: Wie kann die Qualität nach der Höhe des Umsatzes gemessen werden?

BAG: Die Qualität der Leistung wird nicht aufgrund des Umsatzes gemessen. Die Frage ist, was geschieht mit einem Patienten mit einer onkologischen Diagnose, der eine bestimmte Operation ambulant durchführen lässt und eventuell sechs Monate später mit Komplikationen hospitalisiert werden muss. Diese Daten interessieren nicht nur die medizinische Welt, sondern auch die Patienten.

FMH: Will die Verwaltung von jedem Patienten wissen, zu welchem Arzt er hingeht? Ist der Patient selbst überhaupt noch anonym?

BAG: In den Spitälern gibt es seit 15 Jahren eine gut geführte Statistik. Man kennt den Prozentsatz der eigenen Patienten, die rehospitalisiert werden. Ein Identifikationscode, der anonymisiert ist, gibt Aufschluss, um welchen Patienten es sich handelt. Es interessiert nur die Diagnose und die Behandlung durch verschiedene Leistungserbringer und nicht der Name des Patienten. Der Datenschutz der betroffenen Patienten kann technisch garantiert werden.

FMH: Bis jetzt ist klar, dass die Daten 2015 nicht vom BFS ans BAG weitergegeben werden. Was genau sieht Art. 59a KVG und die dazugehörende Verordnung mit dem Datensatz 2016 vor? Wer bekommt diesen Datensatz und in welcher Form?

BAG: In den entsprechenden publizierten Verordnungen ist die Verwendung klar umschrieben. Vorgesehen ist, dass die Daten nur aggregiert und keine Einzeldaten an die Versicherer weitergegeben werden. Das BAG bekommt Einzeldaten zu Personalbestand, das Angebot an Aus- und Weiterbildungsplätzen wie auch die Leistungsdaten pro Leistungserbringer (juristische Person). Die Personaldaten (Ärzte) als Einzeldaten werden anonymisiert zugestellt. Die jetzt erfassten Daten für das Jahr 2015 werden nicht weitergegeben, weder an das BAG noch an die Versicherer.

Jürg Schlup/Präsident FMH bedankt sich bei den Gästen für die Bereitschaft, an der heutigen ÄK teilzunehmen und die Fragen der Ärzteschaft zu beantworten. Die Delegierten führen nach der Verabschiedung ihre Diskussion weiter.

Christoph Bosshard/ZV FMH geht nochmals auf die in diesem Jahr startende Datenerhebung der Daten 2015 ein und hält fest, dass mit dem BFS betreffend Nutzung ausschliesslich zu statistischen Zwecken noch die vertragliche Situation geregelt wird. Art. 22 des BStatG sieht eine Busse vor, wer falsche oder unvollständige Angaben liefert. Die Wegbedingung von Sanktionen wird ebenfalls Teil des Vertrags mit dem BFS sein.

RA Michel Meier präzisiert, dass zwischen den beiden Erhebungen unterschieden werden muss. Die erste Erhebung folgt einer anderen gesetzlichen Grundlage. Nach den heutigen Voten des BAG und des BFS hat die FMH die grösstmöglichste Sicherheit erhalten, dass diese nur statistischen Zwecken dient. Es ändert sich letztlich nichts daran, dass die erste Erhebung genau gleich obligatorisch ist wie die noch folgenden. Das Sanktionswesen wurde jedoch erheblich relativiert. Auf die Frage, wie die statistischen Auswertungen gerade bei polymorbiden Patienten beeinflusst werden können, verweist Christoph Bosshard/ZV FMH auf die in der SÄZ Nr. 41/2016 publizierten Empfehlungen «Gesundheitsbezogene Register - gemeinsame Empfehlungen». Betreffend die Anonymisierung der Daten unterstreicht er, dass die Frage, für welchen gesetzlichen Zweck es welche Anonymisierung braucht, noch nicht geklärt ist. Bevor das Datenbearbeitungsreglement nicht finalisiert ist, können keine Daten basierend auf KVG geliefert werden. Wie die Vertreter des BAG und BFS heute bestätigt haben, werden die jetzt erhobenen Daten auf Basis des Bundesstatistikgesetzes verwendet. Die Daten dieser ersten Erhebung werden weder heute noch in Zukunft an das BAG weitergegeben.

Die Anwesenden sind sich einig, dass die Schwierigkeit nicht die Datenerfassung, sondern die anschliessende Interpretation darstellt. Der Ärzteschaft ist es kaum möglich, zu öffentlichen Anschuldigungen Stellung zu beziehen. Deshalb ist es zwingend, dass die Ärzteschaft Einblick in die erhobenen Daten nehmen kann und bei der der Festlegung bzw. Validierung von Qualitätsindikatoren einbezogen wird.

Urs Stoffel/ZV FMH erwähnt das Projekt «Monitoring», welches der ZV am 20.10.2016 verabschiedet und das Departement Ambulante Versorgung und Tarife mit dem Aufbau mandatiert hat. Die Ärzteschaft wird immer wieder mit überraschenden Mitteilungen seitens Versicherer und BAG konfrontiert, die den Ärzten eine Mengenausweitung und damit die Verantwortung für die Kostenentwicklung zuschieben. Innert kürzester Zeit muss das zuständige Departement Analysen, Argumente, Begründungen und Interpretationen für die von fremder Seite erstellten Statistiken zu den Kostenentwicklungen bereitstellen. Die FMH ist damit immer wieder in die klassische Verteidigungsrolle gedrängt und kann nur reagieren, statt proaktiv zu handeln. Mit dem Datenpool von NewIndex steht eine umfangreiche und nütz- 
liche Datenbasis zur Verfügung. Der Aufbau des FMHinternen Monitorings zur Tarifanwendung geschieht in Zusammenarbeit mit NewIndex und der Ärztekasse. Jürg Schlup/Präsident FMH hält fest, dass der ZV FMH die Mitglieder regelmässig über den aktuellen Stand informieren und auch in die weiteren Diskussionen einbeziehen wird.

Nach den Diskussionen stellt Hans-Anton Vogel/Präsident Appenzeller Ärztegesellschaft folgenden Antrag:

Antrag Vogel:

Die Datenlieferung erfolgt erst, wenn die Reglemente bekannt sind. Die Daten werden der FMH zur Verfügung gestellt.

Beschluss:

Da deutlich weniger als die Hälfte der Ärztekammerdelegierten abwesend sind, kann über den Antrag aufgrund des fehlenden Quorums nicht abgestimmt werden.

\section{IPI: Beendigung Leistungsvertrag}

Anne-Geneviève Bütikofer/GS FMH rapportiert über die Beendigung des Leistungsvertrags. Die ÄK hat im Oktober 2013 einen Sonderbeitrag fürs IPI unter der Auflage genehmigt, dass ein Leistungsvertrag zwischen der FMH und dem IPI abgeschlossen wird. Im Februar 2014 wurde dieser unterzeichnet. Die ÄK hat im Oktober 2014 den Leistungsvertrag einzig um ein Jahr verlängert und entschieden, das IPI 2016 in das Departement eHealth zu integrieren. An der ÄK vom 29.10.2015 zeigte sich jedoch, dass eine Integration ins Departement eHealth gescheitert war. Die ÄK hat dann das Departement beauftragt, die Praxisinformatikthemen über Mandate ab 2016 zu übernehmen. Das Generalsekretariat hat zwischen Oktober 2015 - März 2016 den Jahresabschluss des IPI überprüft und bis Juli 2016 eine Auflösungsvereinbarung verhandelt. Die FMH hat im Geschäftsjahr 2015 dem IPI einen Betrag von CHF 519000 entrichtet. Dieser Betrag beinhaltet neben den Basisinfrastrukturen auch die Leistungsentschädigung. Die FMH verzichtete auf die Rückerstattung der nicht benützten Entschädigung von CHF 21730 sowie auf die Rückzahlung des Mobiliars. Das IPI hat für 2016 auf eine Entschädigung der Arbeiten der IHAMZ wie auch auf weitere Spesenforderungen des IPI-Präsidenten verzichtet. Die vom IPI nicht korrekt verrechneten MwSt-Rechnungen von 2015 im Betrag von CHF 42000 wurden von der FMH beglichen. Alle Ergebnisse und Unterlagen sind Eigentum der FMH. Die FMH räumt dem IPI jedoch an den Ergebnissen das Recht zur nicht ausschliesslichen Nutzung und Weiterentwicklung ein. Jede Partei verzichtet gegenüber der anderen Partei auf eine Geltendmachung von ausschliesslichen
Nutzungsrechten oder auf Massnahmen zur Durchsetzung von behaupteten alleinigen Nutzungsrechten an diesen erarbeiteten Ergebnissen. Die Werkqualität dieser Ergebnisse wird gegenseitig anerkannt. Der Saldo als Rückstellung zugunsten der Praxisinformatik beträgt per 31.12.2016 CHF 670 000. Mit der Auflösungsvereinbarung vom 16.8.2016 sind die FMH und das IPI per Saldo aller gegenseitigen Ansprüche auseinandergesetzt. Es bestehen keine gegenseitigen Ansprüche.

\section{Informationen aus ZV, General- sekretariat, SIWF und Abteilungen}

Den Delegierten wurden die schriftlichen Berichte aus den Departementen und dem Generalsekretariat anlässlich der ÄK verteilt.

\subsection{TARPSY und ST-Reha: mündliche Informa- tionen}

Jürg Unger-Köppel/ZV FMH informiert über die neuen Tarifsysteme TARPSY für die stationäre Psychiatrie und ST-Reha für die Rehabilitation. TARPSY für die Erwachsenen wird auf den 1.1.2018 eingeführt. Für die Kinder- und Jugendpsychiatrie ist die Einführung frühestens per 1.1.2019 vorgesehen, da die Datenqualität zur Kalkulation der Tarifstruktur noch ungenügend ist. Ein Einführung von ST-Reha wurde verschoben und ist für den 1.1.2020 geplant. Im Gegensatz zu DRG wurden TARPSY und ST-Reha in der Schweiz entwickelt. Die Leistungen der stationären Psychiatrie sollen schweizweit mit einem einheitlichen leistungsbezogenen Tarifsystem vergütet werden. Jürg Unger-Köppel weist darauf hin, dass die FMH bei der Gestaltung der Tarifstruktur eng miteinbezogen wurde und die $\mathrm{Zu}-$ sammenarbeit mit den Partnern der SwissDRG AG sowie die Kooperation beispielsweise mit $\mathrm{H}+$ gut funktioniert. Weitere Informationen können unter tarife[at] fmh.ch bezogen werden.

\section{Varia}

Anne-Geneviève Bütikofer/GS FMH gibt die Sitzungsdaten der ÄK für 2017 und 2018 bekannt. 2017 finden diese am 4.5. und 26.10. und 2018 am 3.5. und 25.10. statt.

Jürg Schlup/Präsident FMH dankt den Delegierten für die engagierte Sitzungsarbeit und ihren täglichen Einsatz für das Gesundheitswesen. Einen besonderen Dank spricht er den Mitarbeitenden der FMH für die Organisation dieser ÄK aus und wünscht allen eine gute Heimreise.

Bildnachweise

Fotos: Frederike Asael 


\begin{tabular}{|c|c|}
\hline Abkürzung & Erläuterung \\
\hline AGZ & Ärztegesellschaft Zürich \\
\hline ÄK & Ärztekammer \\
\hline AL & Ärztliche Leistungen \\
\hline BAG & Bundesamt für Gesundheit \\
\hline BFI-Botschaft & Botschaft zur Förderung von Bildung, Forschung und Innovation \\
\hline BFS & Bundesamt für Statistik \\
\hline BR & Bundesrat \\
\hline BStatG & Bundesstatistikgesetz \\
\hline DV & Delegiertenversammlung \\
\hline DRG & Diagnosis Related Groups \\
\hline EDI & Eidg. Departement des Innern \\
\hline EDÖB & Eidg. Datenschutz- und Öffentlichkeitsbeauftragter \\
\hline EMH & Schweizerischer Ärzteverlag \\
\hline $\mathrm{fmCh}$ & foederatio medicorum chirurgicorum helvetica \\
\hline $\mathrm{FMH}$ & Verbindung der Schweizer Ärztinnen und Ärzte \\
\hline GPK & Geschäftsprüfungskommission \\
\hline GO & Geschäftsordnung \\
\hline GS & Generalsekretärin \\
\hline $\mathrm{H}+$ & Die Spitäler der Schweiz \\
\hline IPI & Institut für Praxisinformatik \\
\hline KVG & Krankenversicherungsgesetz \\
\hline KVV & Krankenversicherungsverordnung \\
\hline MedReg & Medizinalberuferegister \\
\hline NAKO & Nationale Konsolidierungsstelle \\
\hline NFP & Nationales Forschungsprogramm \\
\hline ОМст & Ordine dei Medici del CantoneTicino \\
\hline RA & Rechtsanwalt \\
\hline SÄZ & Schweizerische Ärztezeitung \\
\hline SAQM & Schweizerische Akademie für Qualität in der Medizin \\
\hline SFSM & Swiss Federation of Specialities in Medicine \\
\hline SHK & Schweizerische Hochschulkonferenz \\
\hline SMSR & Société médicale de la Suisse romande \\
\hline SIWF & Schweizerisches Institut für ärztliche Weiter- und Fortbildung \\
\hline RoKo & Rollende Kostenstudie \\
\hline TARMED & Tarif Medizin für ambulante Einzelleistungen \\
\hline TARPSY & Tarifstruktur für die stationäre Psychiatrie \\
\hline ST-Reha & Tarifstruktur für die stationäre Rehabilitation \\
\hline VEDAG & Verband deutschschweizerischer Ärztegesellschaften \\
\hline VSAO & Verband Schweizerische Assistenz- und Oberärzte/-innen \\
\hline ZV & Zentralvorstand \\
\hline
\end{tabular}

